\title{
Diagnosing simultaneous faults using the local regularity of vibration signals
}

\author{
Juhani Nissilä and Jouni Laurila \\ Intelligent Machines and Systems, Faculty of Technology, \\ P.O. BOX 4200, FI-90014, University of Oulu, Finland \\ E-mail: juhani.nissila@oulu.fi, jouni.laurila@oulu.fi
}

\begin{abstract}
The regularity of the vibration signals measured from a rotating machine is often affected by the condition of the machine. The fractional order of regularity can be measured using the definition of Hölder continuity. In this paper, we review the connection between the pointwise Hölder regularity of a signal and its wavelet transform. We calculate the wavelet transform modulus of acceleration measurements from a test rig. The effects of different faults were recorded, such as unbalance, the coupling misalignment of a claw clutch, the absence of lubrication in a ball bearing, the absence of the bearing's cage, and their combinations. An analysis of the estimated isolated pointwise regularities from the wavelet transform modulus maxima ridges shows that the faults often cause irregularities in the signals and that their locations and frequencies can be used in diagnosing the faults. Coupling misalignment and the absence of lubrication in a ball bearing both cause impact-like vibrations, but these impacts have positive and negative regularities in the case of a coupling misalignment and mainly negative in the case of a dry bearing. Unbalance is best diagnosed from the integrals of the acceleration signals using traditional methods. In diagnosing the misalignment, bearing problems and simultaneous faults, the local regularity analysis outperforms the use of high order norms of differentiated acceleration measurements (i.e. jerk and snap signals). Using just three features (the number of local irregularities in an acceleration signal, their mean Hölder regularity and the arithmetic mean of the absolute values of a velocity signal), a quadratic classifier can be constructed whose estimated classification error is only $0.3 \%$.
\end{abstract}

Keywords: Hölder regularity, wavelet transform modulus maxima, coupling misalignment, bearing diagnostics, unbalance, vibration measurements 


\section{Introduction}

The task of diagnosing simultaneously occurring faults in rotating machinery is challenging. Some faultrelated signals are easily masked by noise or the vibration caused by more severe faults. One way of approaching this problem is to decompose the signal into a finite number of components that convey different kinds of diagnostic information. In [1], the vibrations caused by inner and outer race faults in a bearing as well as wear and snaggletooth in a gear are separated using variation mode decomposition (VMD) and a 1.5-dimension envelope spectrum. In [2], a broken gear tooth and a tooth crack together with an outer race defect are diagnosed using resonancebased signal sparse decomposition (RSSD). Another useful tool for vibration signal decomposition and fault-related feature reconstruction is singular value decomposition (SVD), which is utilised in [3] to diagnose inner and outer race faults in a bearing. The second approach to compound fault diagnosis is to find and calculate features that correlate well with different kinds of faults and then use the features for classification. This paper studies the second approach. A thorough study of classical features related to differentiation, integration, norms and statistical moments is provided in [4].

In the vibration measurements of rotating machines, the highest relative change between a faulty state and a normal operating condition often occurs when the vibration signal's fractional order of derivative is a real number. In [5], it was suggested that this might be due to the reduced regularity of the vibration signal. The authors of [5] used the signal's wavelet transform to estimate its isolated local irregularities and showed that the locations of the negative Hölder regularities corresponded to points where the rolling elements hit the faults on the inner race of a slowly rotating bearing. The largest negative regularities were -5 , and the defects also caused an increase in the number of positive Hölder exponents, which were mainly between 0.5 and 1.5. The bearing in normal condition did not cause any negative Hölder exponents. The mathematical background for using the continuous wavelet transform for the regularity analysis is mainly based on the work of Jaffard [6] and Mallat and Hwang [7]. We abbreviate the continuous wavelet transform as CWT, its modulus as WTM (wavelet transform modulus) and the wavelet transform modulus maxima as WTMM. The definitions of these mathematical objects are presented in section 2 .

The mathematical theory is sophisticated and not easy to implement in digital vibration measurements. A survey of previous technical articles reveals the pitfalls that one may encounter. In [8], [9] and [10], for example, the Hölder exponent is estimated at every point of the digital signal by simply using all the corresponding wavelet values at different scales. The problem here is that the modulus maxima ridges are not identified and it is assumed that pointwise regularity can be meaningfully computed at each point. The maxima ridges (which are clearly visible in the CWT of most vibration measurements) often spread out as the scales increase. This is why it is necessary to study the wavelet values in the cone of influence of the point of interest. Moreover, if there are parts in the signal where the estimated CWT values are almost zero at small scales (these appear between the maxima ridges), the signal there is uniformly Hölder continuous. Since we suppose that the isolated irregularities carry the diagnostic information that we are after, it might be a good idea to ignore these smoother areas.

Despite these shortcomings in mathematics, it was at least shown in [8] that the CWT can reveal the small amplitude rattle in a structure mounted on non-symmetric bumpers and subjected to harmonic excitation. In [9], CWT-based methods were capable of indicating the progression of a gear fault. In [10], Hölder regularity estimation is used in diagnosing the inner and outer race faults in a bearing, though the signal processing is so obscure that the validity of the results is unclear. The vibration signals even in normal conditions show negative regularities of order -34 , which would be very irregular. All of these three papers $[8,9,10]$ also share the same error in the wavelet theory, in that they define the CWT so that the $L^{2}$ norm of the signal is normalised, but the formulas describing the decay of the CWT and Hölder regularity are taken from articles where the $L^{1}$ norm is normalised. This gives an additional error of size $1 / 2$ in all of the estimated regularities. This error is also present in [11], in which gear faults of different severity are studied. The main result in [11] is that the mean Hölder exponents of a group of signals decrease as the condition of the gears deteriorates.

There is one more caveat here, since not all the WTMM ridges indicate an isolated irregularity. If our wavelet has $n$ vanishing moments, there may be ridges in the CWT that seem to indicate an isolated irregularity with the Hölder exponent $n$. However, as indicated by the review of the theory in section 2, only isolated irregularities with exponent $\mu<n$ can be verified. In the case there are ridges where the estimated Hölder exponent maxes out to $n$, one should compute the CWT again using a wavelet with more than $n$ vanishing moments in order to see what is going on. The signal may even be infinitely differentiable at those points. In [12], different faults in a test rig are analysed and almost sinusoidal signals are recorded in the case of unbalance. The CWT of one of these 
signals shows maxima lines where the estimated Hölder exponents vary between 1.8017 and 1.9564. Since the wavelet that was used has two vanishing moments, some of the points may in fact be much more regular. Here we note that only one decimal point of accuracy is typically achieved with numerical calculations of this kind. It was observed in [12] that an oil whirl caused regularities between 1.0 and 1.8, a coupling misalignment regularities between 0.3 and 1.8, and a rub impact between rotor and stator regularities between -0.5 and 1.3. In all of the cases the smallest Hölder exponents were observed when the fault was more severe.

There are also other ways of using the continuous wavelet transform in condition monitoring. In [13], the CWT is viewed as a time-frequency representation of the measured vibration signal, and diagnostic information on bearing defects in a test rig is based on features derived from the vibrations near the system's resonance frequency $2000 \mathrm{~Hz}$ or in a wider band from $1000 \mathrm{~Hz}$ to $8000 \mathrm{~Hz}$. The vibration signals in [14] go through a signal decomposition process after which the fault-related signal's spectrum is computed using the wavelet analysis. In [15], CWT is one of the signal processing methods compared for bearing diagnostics. They use a time slice of the CWT near the resonance frequency for detecting inner and outer race bearing defects. Fortunately, the 3D plots of the CWT coefficients are also plotted in the paper and we can use them to see that the width of the defect correlates with the irregularity of the vibration. In the case of an outer race defect, a defect of width $500 \mu \mathrm{m}$ causes a CWT where the coefficients increase towards bigger scales, so it has irregularities with positive Hölder exponents. Defects of width $1000 \mu \mathrm{m}, 1500 \mu \mathrm{m}$ and $2000 \mu \mathrm{m}$ cause vibrations in which the CWT coefficients increase towards small scales and more steeply with wider defects. This is one evidence more for the observation that bearing defects easily cause negative irregularities.

Different gear tooth failures were diagnosed in [16] using WTMM ridges. The estimated local Hölder exponents had a mean value of 1.538 in the case of a tooth crack and 0.968 in the case of a completely lost tooth. Interestingly, the exponent was independent of the loading conditions and also of the depths of the crack (which were 15\%,33\%, 50\% and $75 \%$ of the tooth root). However, the depth of the crack was related to the height of the WTMM ridges. These observations are all very useful for diagnostic purposes. This kind of diagnostic potential is ignored in [17], where the authors state that: "...we are not interested in the Lipschitz exponent value of singularity but the occurrence and position of it". They analyse test runs where simple helical gears are driven to tooth failure. Using just the position and the number of maxima lines, they are able to show when severe failures start to appear.

WTMM ridges were also utilised in [18] to filter vibration signals. Only the ridges that propagated long enough with an increasing scale were utilised in reconstructing the signal. This procedure amplified the low frequency impacts caused by the bearing's inner and outer race faults. The authors argue for this filtering as follows: "... magnitude of the maxima corresponding to noise remains as smaller values and does not propagate to larger scales. Furthermore, singularities caused by high frequency structural resonance also do not propagate to large scales by the very nature of scale s being inversely proportional to the frequency". However, although the results are encouraging, statements of this kind are inaccurate. According to other studies we have just reviewed, negative regularities can occur with bearing faults, and the maxima lines corresponding to them can decay to very small values as $s$ increases.

In [19], the first author of this paper studied acceleration measurements of the epicyclic gearboxes of a hydroelectric power station. The main observation was that the vibrations caused by the higher speed gearbox were more irregular. Discrete Fourier transforms of the estimated pointwise regularities were computed, indicating that the periodic variations in the irregularities were caused by gear mesh events in both the gearboxes and also by the rotating turbine blades in the slower speed gearbox.

To sum up our observations from previous studies, it seems that often either just the Hölder exponents or the locations of the irregularities are examined in detail. In addition, the height of the WTMM ridges is rarely considered, even though it can easily be characterised using a constant calculated simultaneously with the Hölder exponent. This paper shows that these constants can also be used to help ignore the computational errors in the CWT. We will combine all the information to separate different simultaneously occurring faults. We will also examine integrated acceleration signals, as unbalance is much easier to diagnose from them than directly from the acceleration measurements.

We investigate the acceleration measurements from a small test rig in its normal condition and in the presence of different faults, which are unbalance, coupling misalignment, the absence of lubrication in a ball bearing, the absence of a bearing cage, and their combinations. In section 2 we present the signal processing methods, i.e. Fourier transforms, the wavelet theory and most importantly the modulus maxima ridges of the continuous wavelet transform for pointwise Hölder regularity estimation of isolated irregularities. The 
test rig and the vibration measurements are presented in section 3 . The numerical analysis of the measurements is presented in section 4. The local regularity analysis is complemented with simple features calculated from integrated and differentiated acceleration measurements, and the various features are compared with pattern recognition algorithms based on their capability to separate the various fault cases. Finally the results are discussed in section 5 .

\section{Signal processing theory}

\subsection{Fourier transforms and derivatives}

The spectrum of the sampled signal $\boldsymbol{x}=\left(x_{0}, \ldots, x_{N-1}\right)$ of length $T=\Delta t \cdot N$ is calculated with the Discrete Fourier transform (DFT)

$\mathcal{F}\{\boldsymbol{x}\}_{k}=X_{k}=\frac{1}{N} \sum_{n=0}^{N-1} x_{n} e^{-i 2 \pi k n / N}$,

and the inverse transform (IDFT) returns the signal at the sample points [20]

$\mathcal{F}^{-1}\{\boldsymbol{X}\}_{n}=x_{n}=\sum_{k=0}^{N-1} X_{k} e^{i 2 \pi k n / N}$.

The cyclic convolution of two sampled signals is

$(\boldsymbol{x} * \boldsymbol{y})_{n}=\sum_{l=0}^{N-1} x_{l} y_{n-l}$,

and its DFT is [20]

$\mathcal{F}\{(\boldsymbol{x} * \boldsymbol{y})\}_{k}=N X_{k} Y_{k}$.

Note that in all the previous equations the sampled signal is assumed to be $N$-periodic. The DFT and IDFT pair can be used for the numerical differentiation and integration of signals. An algorithm for this consists of calculating the DFT coefficients $X_{k}$ and then forming a new sequence $G_{k}, 0 \leq k \leq N-1$, with $G_{0}=0$ (here we assume that the mean of the signal is zero) and

$$
\begin{aligned}
G_{k} & =\left(\frac{2 \pi k i}{T}\right)^{z} X_{k}, & & 0<k<N / 2 \\
G_{N+k} & =\left(\frac{2 \pi k i}{T}\right)^{z} X_{N+k}, & & -N / 2<k<0 \\
G_{N / 2} & =\left(\frac{\pi N}{T}\right)^{z} \cos \left(z \frac{\pi}{2}\right) X_{N / 2} & & \text { (if } N \text { is even), }
\end{aligned}
$$

where $z$ is the order of derivative (or integral when negative). Finally we obtain the vector $\boldsymbol{x}^{(z)}$ with the IDFT

$$
x_{n}^{(z)}=\mathcal{F}^{-1}\{\boldsymbol{G}\}_{n} .
$$

The only problematic part in deriving algorithm (5) is the term $G_{N / 2}$ for even $N$, which the authors presented in [21]. The algorithm also works with any complex $z$, in which case we use the principal values of $\left(\frac{2 \pi k i}{T}\right)^{z}$ and $\left(\frac{\pi N}{T}\right)^{z}$. It is then used to calculate an approximation of the Fourier or Weyl fractional derivatives and integrals. This operation is also sometimes called differintegration.

The DFT assumes that the sequence is periodic, so it is thus practical to window the signal to attenuate any discontinuities at the end points of the sequence. Since we are interested in the irregularities of the signal, we want our window function to be smooth so that it does not introduce any new irregularities. We use the Planck-taper window originally defined for gravitational wave detection in [22]

$$
w_{1}(t)= \begin{cases}0, & \text { if } t \leq 0 \\ (Z(t)+1)^{-1}, & \text { if } 0<t<\epsilon T \\ 1, & \text { if } \epsilon T \leq t \leq T / 2 \\ w(T-t), & \text { if } t>T / 2,\end{cases}
$$

where $\epsilon$ is the portion of the signal for ascent and descent and

$$
Z(t)=\frac{\epsilon T}{t}+\frac{\epsilon T}{t-\epsilon T} .
$$

The discretised version of (6) to $N$ samples is

$$
w_{n}= \begin{cases}0, & \text { if } n \leq 0 \\ \left(Z_{n}+1\right)^{-1}, & \text { if } 0<n<\epsilon(N-1) \\ 1, & \text { if } \epsilon(N-1) \leq n \leq N / 2 \\ w_{N-n}, & \text { if } n>N / 2,\end{cases}
$$

where

$$
Z_{n}=\epsilon(N-1)\left(\frac{1}{n}+\frac{1}{n-\epsilon(N-1)}\right) .
$$

A similar function was also introduced in [23] using integrals in its definition

$w_{2}(t)= \begin{cases}0, & \text { if } t \leq 0 \\ \frac{1}{E} \int_{0}^{t} e^{(\tau(\tau-T / \epsilon))^{-1}} \mathrm{~d} \tau, & \text { if } 0<t<T / \epsilon \\ 1, & \text { if } T / \epsilon \leq t \leq T / 2 \\ w_{2}(T-t), & \text { if } t>T / 2 .\end{cases}$

Here $E=\int_{0}^{T / \epsilon} e^{(\tau(\tau-T / \epsilon))^{-1}} \mathrm{~d} \tau$. One must approximate the integrals numerically with the trapezoidal rule, for example, to obtain the sampled version of this window.

The continuous Fourier transform $\hat{x}$ of $x$ is

$\mathcal{F}\{x\}=\hat{x}(\nu)=\int_{-\infty}^{\infty} x(t) e^{-i 2 \pi \nu t} \mathrm{~d} t$.

Its inverse transform is

$\mathcal{F}^{-1}\{\hat{x}\}=\int_{-\infty}^{\infty} \hat{x}(\nu) e^{i 2 \pi \nu t} \mathrm{~d} \nu$. 
Scaling the signal results in a transform

$\mathcal{F}\{x(t / s)\}=|s| \hat{x}(s \nu)$,

and the convolution

$(x * y)(t)=\int_{-\infty}^{\infty} x(\tau) y(t-\tau) \mathrm{d} \tau$,

is transformed into multiplication

$\mathcal{F}\{(x * y)\}=\hat{x}(\nu) \hat{y}(\nu)$.

Equations (11) and (12) are valid if, for example, $x, y \in L^{2}(\mathbb{R})$, i.e. they are square integrable.

\subsection{Continuous wavelet transform}

Wavelet is informally defined as a brief oscillation. Thus it either has compact support or its amplitude at the infinities decays to zero sufficiently quickly. For the context of a regularity analysis, we also suppose that the wavelet $\psi$ is real-valued. An admissible wavelet's Fourier transform satisfies

$\int_{-\infty}^{\infty} \frac{|\hat{\psi}(\nu)|^{2}}{|\nu|} \mathrm{d} t<\infty$.

We want to dilate the wavelet by $s>0$ but retain its $L^{1}$ norm

$\psi_{s}(t)=\frac{1}{s} \psi\left(\frac{t}{s}\right)$.

The wavelet transform of $x$ is then

$$
\begin{aligned}
W x(s, t) & =\left(x * \psi_{s}\right)(t) \\
& =\frac{1}{s} \int_{-\infty}^{\infty} x(\tau) \psi\left(\frac{t-\tau}{s}\right) \mathrm{d} \tau
\end{aligned}
$$

Here $t$ is the point of interest in the signal and $s$ is the positive scale at which the wavelet is dilated. The CWT is often defined without mirroring the wavelet, but we define it like this in order to utilise the simple convolution equivalence. The reconstruction of the original function is possible in suitable function spaces if the admissibility condition is satisfied, see [24], for example. To account for the possible locally singular behaviour of vibration measurements, $x$ must be from the class of generalised functions called tempered distributions [25]. We do not cover the theory of generalised functions here, however, but only note that (15) is well defined if $x$ is a tempered distribution of order $m$ and if the wavelet $\psi$ is $m$ times continuously differentiable. The Fourier transform maps a tempered distribution into a tempered distribution and the inverse reconstructs the original signal.

The Fourier transform of the CWT at a fixed scale $s$ is easily computed with (10) and (12)

$\mathcal{F}\{W x(s, t)\}=\hat{x}(\nu) \hat{\psi}(s \nu)$,

A wavelet has $m$ vanishing moments if

$\int_{-\infty}^{\infty} t^{k} \psi(t) \mathrm{d} t=0, \quad$ for $k=0,1, \ldots, m-1$.
In practice, the CWT is estimated from a finite sequence using summations instead of integrals. The word 'continuous' is still used here to separate the definition from the actual discrete wavelet transform, where the discrete scales are sampled as powers of two, and the discrete wavelets at these scales form an orthogonal set. In this paper, we utilise the DFT to calculate the estimates for the CWT. The wavelet that we use in the numerical calculations is the second derivative of a central $B$-spline and its DFT is

$B_{k}=\frac{1.83}{N}\left(\frac{i 2 \pi k}{N}\right)^{2}\left|\frac{\sin (2 \pi k / N)}{2 \pi k / N}\right|^{5}$,

$B_{N / 2}=\frac{1.83}{N} \pi^{2} \cos (\pi)\left|\frac{\sin (\pi)}{\pi}\right|^{5}=0$,

where $-N / 2<k<N / 2$ (because of $N$-periodicity, the negative frequencies correspond to $N / 2<k<N$ in our definition of the DFT). It has compact support and two vanishing moments. The constant 1.83 is experimentally chosen so that that the $l^{1}$ norm of the IDFT of $\boldsymbol{B}$ is approximately 1 . Note that the numerical differentiation has been carried out with the algorithm (5) using the scaling $\Delta t=1, T=\Delta t \cdot N=N$. Time domain representations of integer and fractional order central B-splines can be found in [26]. The wavelet is visualised in figure 1 with $N=256$ and $s=6$. Also note that it is centered around the point $n=0$ and it is $N$-periodic. The value $N=32768$ will be used in the calculations. The wavelet will then have a longer interval of almost zero values in the middle, but is in other aspects identical to figure 1.

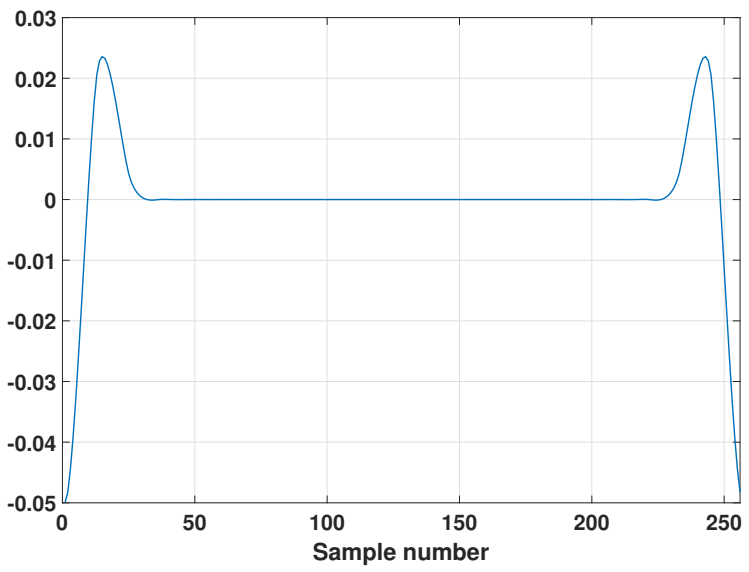

Figure 1. The wavelet $\mathcal{F}^{-1}\{\boldsymbol{B}\}$ of length $N=256$ when $s=6$.

Let us denote the estimated CWT at scale $s$ by $W \boldsymbol{x}_{s, n}$. To calculate its DFT, we first window the measured signal $\boldsymbol{x}$ with (7), i.e. compute $x_{n} w_{n}$, calculate its DFT $\mathcal{F}\left\{x_{n} w_{n}\right\}_{k}$ and then estimate (16) using its DFT analogue (4)

$\mathcal{F}\left\{W \boldsymbol{x}_{s, n}\right\}_{k}=N \mathcal{F}\left\{x_{n} w_{n}\right\}_{k} B_{s k}$. 
Finally we obtain $W \boldsymbol{x}_{s, n}$ at the desired scales using the IDFT.

If the signal is bounded, we can also bound the CWT using a special case of Hölder's inequality

$$
\begin{aligned}
|W x(s, t)| & \leq \sup _{t \in \mathbb{R}}(x(t)) \int_{-\infty}^{\infty}\left|\psi_{s}(t)\right| \mathrm{d} \tau \\
& =\|x\|_{\infty} \cdot\|\psi\|_{1} .
\end{aligned}
$$

Corresponding equations are naturally also valid for the discrete case

$$
\left|W \boldsymbol{x}_{s, n}\right| \leq\|\boldsymbol{x}\|_{\infty} \cdot\|\boldsymbol{\psi}\|_{1} .
$$

To estimate the numerical error in the discretisation of our chosen wavelet at small scales, we have plotted the computed norms $\left\|\mathcal{F}^{-1}\{\boldsymbol{B}\}\right\|_{1}$ in figure 2 from $s=0.5$ to 6 and using $N=32768$. Since there are more discrete points representing the dilated wavelet at large scales, it is no surprise that the norms are more accurately close to 1 at the right end of the figure. For $s \geq 1.5$ the error in norms is within $2 \%$. When $s<1$, the error may be as much as $16 \%$. Equations (21) and (22) are equalities if the signal is constant. If the signal is not constant, however, they still give an estimate of the numerical error. With the wavelet $\mathcal{F}^{-1}\{\boldsymbol{B}\}$, for example, we see that if we would compute the CWT at the scales $0.5 \leq s<1$, the absolute values of our estimated wavelet coefficients would probably be bounded by either $0.84 \cdot\|\boldsymbol{x}\|_{\infty}$ or $1.16 \cdot\|\boldsymbol{x}\|_{\infty}$ and we would have no information on what the correct bound is.

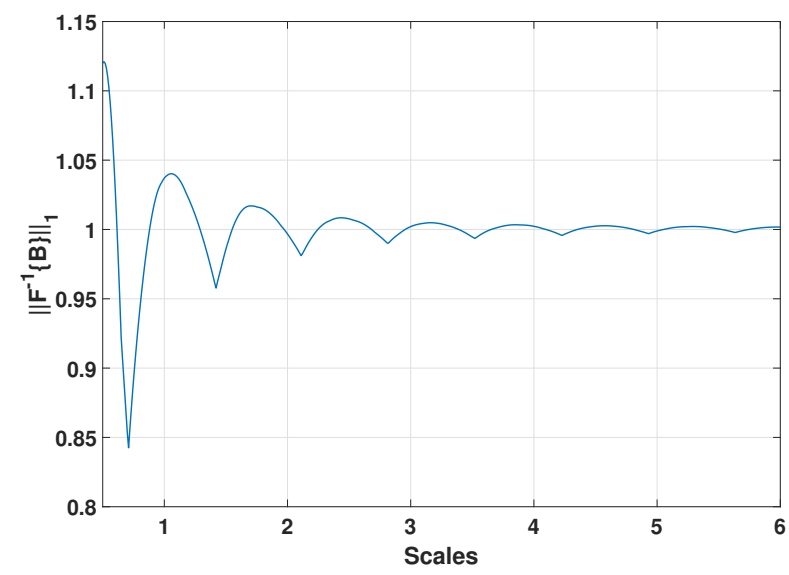

Figure 2. Computed $l^{1}$ norms of the wavelet $\mathcal{F}^{-1}\{\boldsymbol{B}\}$ of length $N=32768$ at different scales. The deviations from the value 1 at small scales are related to discretisation errors.

There is a balance here that must be carefully chosen. The small scales convey detailed information on the local changes of the signal, but we can only zoom in up to some resolution in this discrete analysis. We choose to use scales from 1.5 to 6 in our analysis.

\subsection{Hölder regularity and wavelet modulus maxima}

Let $\mu \geq 0$. A function $x$ is pointwise $\mu$-Hölder continuous at $t_{0}$ if

$\left|x\left(t_{0}+h\right)-P_{m}(h)\right| \leq C|h|^{\mu}$,

for small values of $|h|$ and $P_{m}$ is a polynomial of degree $m \leq \mu$. If $\mu$ is a non-integer, then $P_{m}$ is the $m+1$ first coefficients of the Taylor polynomial of $x$ at $t_{0}$. If for all $t_{0}+h$ on an interval, the condition (23) is satisfied with the same constant $C$, then $x$ is uniformly $\mu$-Hölder continuous on that interval.

The extension of this definition to negative Hölder exponents is not trivial. With the help of generalised functions we can satisfactorily extend it to small intervals. Let $\mu$ be a non-integer. We say that a tempered distribution $x$ of finite order is uniformly $\mu$ Hölder on the interval $] a, b[$ if its primitive is $\mu+1$ Hölder on the same interval (primitive $=$ indefinite integral for $x \in L^{1}(a, b)$, see [25] for tempered distributions).

We wish to find isolated irregularities in signals, i.e. points $t_{0}$ where $f$ is pointwise $\mu$-Hölder and also uniformly $m$-Hölder with $m>\mu$ on that interval when $t_{0}$ is excluded.

When traversing the time-scale half-plane through the scales, a series of connected local maxima or minima in the wavelet transform may be observed. These are called wavelet transform modulus maxima ridges (WTMM ridges). Mallat and Hwang proved in [7] that if there are no such ridges at the fine scales on a given interval, then $f$ is uniformly Hölder continuous on that interval. Therefore, we expect that the modulus maxima ridges will reveal the isolated irregularities in the signal. In fact, we can even read the Hölder exponent from the decay rate of these ridges converging to $t_{0}$ at fine scales if the exponent is noninteger and our wavelet has enough vanishing moments. The next theorem is also proved in [7]. A smoothing function is a non-negative function whose integral over $\mathbb{R}$ equals one.

Theorem 1. Suppose that the admissible wavelet $\psi$ has compact support, is $m$ times continuously differentiable and is the mth derivative of a smoothing function. Let $x$ be a tempered distribution and its wavelet transform be well defined on $] a, b\left[\right.$ and let $t_{0} \in$ ]$a, b\left[\right.$. If there exists a constant $C$ and a scale $s_{0}$ such that all the modulus maxima of $W x(s, t)$ belong to the cone

$\left|t-t_{0}\right|<C s$,

then $x$ is uniformly $m$-Hölder in the neighbourhood of all the points $t \in] a, b\left[, t \neq t_{0}\right.$. Let $\mu<m$ be a noninteger. Then $x$ is $\mu$-Hölder at $t_{0}$ if and only if

$|W x(s, t)| \leq A s^{\mu}$,

at each modulus maxima inside the cone (24). 
The condition that the WTMM ridges are inside the cone of influence of the point of interest is crucial. It is easy to construct functions that oscillate infinitely many times on a bounded interval and for such functions we will have some points where this condition is not satisfied. Although it is possible that such behaviour or something very close to it may exist in real vibration measurements as well, we will assume here that all the irregularities in our measured vibrations are isolated. The irregularities will thus be found using the following algorithm, which is a modified version of the ones used in [5] and [19].

(i) Estimate the CWT with a wavelet that has the desired number of vanishing moments.

(ii) Find the local minima and maxima at each computed scale.

(iii) Follow the minima and maxima from the smallest available scale upwards to register the modulus maxima ridges. The ridges are found by requiring that the connected maximas/minimas spread so that they form at most one discrete sample apart between the scales. We only consider scales that continue uninterrupted from the smallest computed scale to the highest computed scale.

(iv) Also estimate the point where the ridge ends at scale 0 by linear extrapolation in the other direction.

(v) Estimate the regularity using (25) and logarithms

$\log (|W x(s, t)|) \leq \log (A)+\mu \log (s)$

and the slope of a least squares line gives $\mu$. We first compute this using the three smallest scales and then add the higher scales one by one. If the estimated $\mu$ changes by more than $30 \%$, the process is stopped and the previous value kept.

(vi) If multiple lines converge to the same point, choose the smallest value as $\mu$.

Based on the results of figure 2 we used scales from 1.5 to 6 with steps of 0.1 in the numerical calculations. We further validated this range by first testing the algorithm with an artificial signal with known local irregularities shown in figure 3 . The signal is

$\frac{H(t-0.01)}{|t-0.01|^{0.8}}+|t-0.02|^{0.4}+H(t-0.03)$,

where $H$ is the Heavyside step function and we sample this signal with 32768 points on the interval [0,0.04]. Due to the sampling, we do not completely see the singularity at the the point $t=0,01$. This is nevertheless a good approximation of what also seems to happen in real vibration measurements: the signal may be "locally unbounded", although our discrete measurements always have finite values.

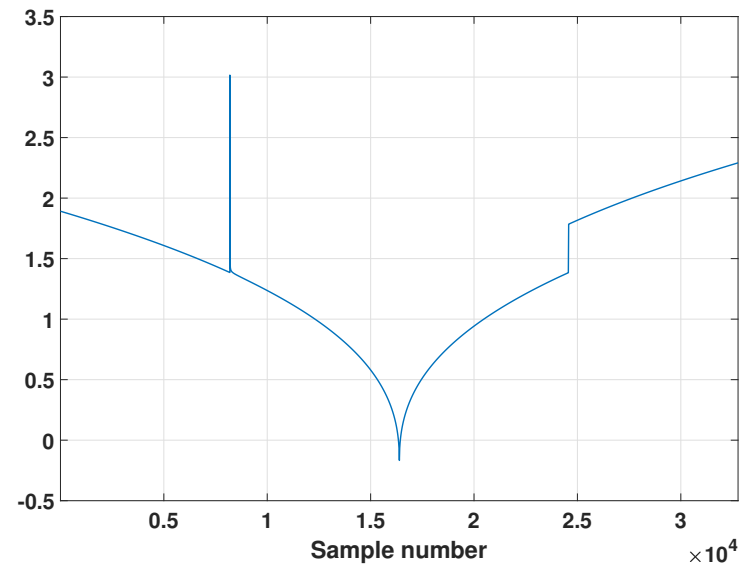

Figure 3. Test signal with regularities $-0.8,0.4$ and 0 from left to right.

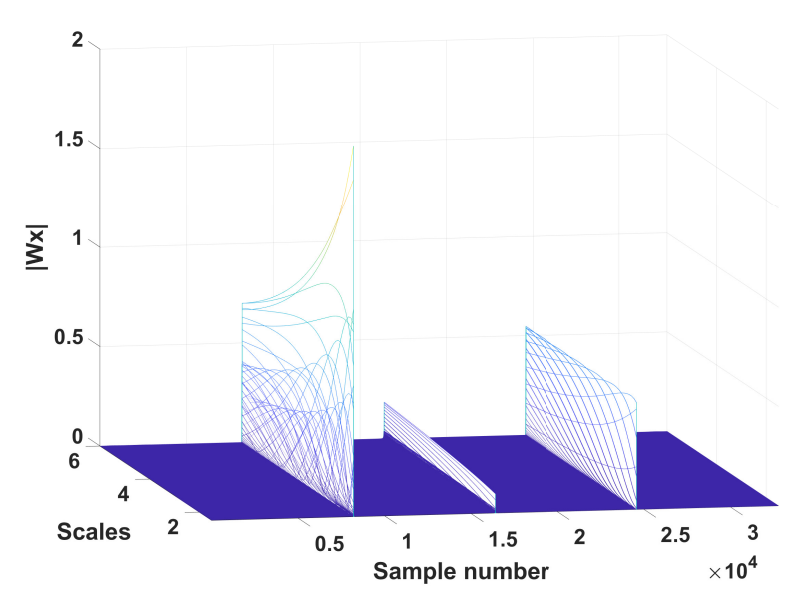

Figure 4. Absolute values of the CWT of the test signal from figure 3.

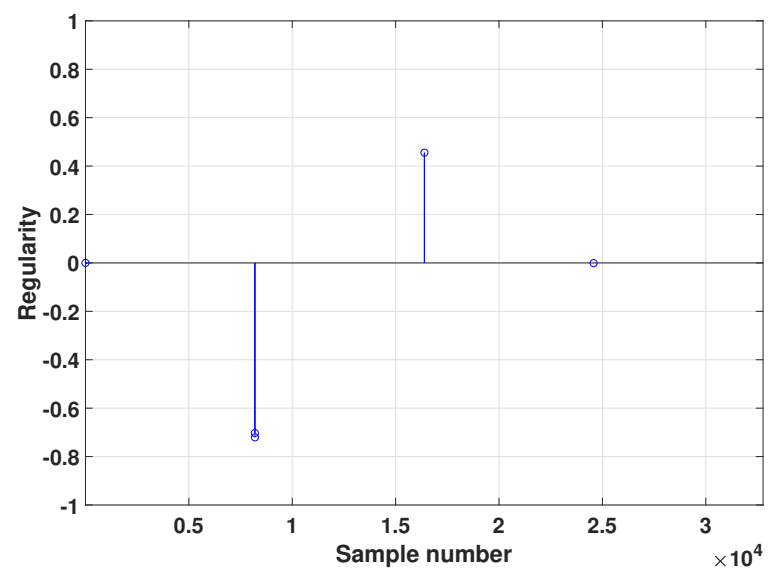

Figure 5. The estimated local regularities and their locations from the CWT of figure 4. 
The estimated CWT of this signal is shown in figure 4. We see that the WTMM ridges behave as predicted by the theory on that range of scales. The estimated Hölder exponents are shown in figure 5. The algorithm may often find several ridges converging near to the same point. The estimated deviate from the correct values by approximately 0.1 .

\subsection{Generalised $l^{p}$ norms, kurtosis and skewness}

The generalised $l^{p}$ norm or Hölder mean of vector $\mathbf{x}^{(z)}$ is

$$
\left\|\mathbf{x}^{(z)}\right\|_{p, \frac{1}{N}}=\left(\frac{1}{N} \sum_{n=0}^{N-1}\left|x_{n}^{(z)}\right|^{p}\right)^{1 / p}
$$

for $p \geq 1$. This is the traditional $l^{p}$ norm with equal weights $1 / N$. The generalisation with respect to cases $-\infty \leq p \leq \infty$ is performed in [27] using the limiting values

$$
\left\|\mathbf{x}^{(z)}\right\|_{p, \frac{1}{N}}= \begin{cases}\left(\sum_{n=0}^{N-1} \frac{1}{N}\left|x_{n}^{(z)}\right|^{p}\right)^{\frac{1}{p}}, & \text { if } p \in \mathbb{R} \backslash\{0\} \\ \left(\prod_{n=0}^{N-1}\left|x_{n}^{(z)}\right|\right)^{\frac{1}{N}}, & \text { if } p=0 \\ \max _{n=0, \ldots, N-1}\left|x_{n}^{(z)}\right|, & \text { if } p=\infty \\ \min _{n=0, \ldots, N-1}\left|x_{n}^{(z)}\right|, & \text { if } p=-\infty .\end{cases}
$$

This definition includes many traditional features, such as minimum value $p=-\infty$, harmonic mean $p=-1$, geometric mean $p=0$, arithmetic mean $p=1$, root mean square (rms) $p=2$ and maximum value (or peak value) $p=\infty$. For $p<0$, all the $x_{n}^{(z)}$ must be non-zero.

Let us denote the expected value of a random variable $x$ by $E(x)$ and its variance by $\sigma^{2}=$ $E\left[(x-E(x))^{2}\right]$. Now we can define unitless statistical features

$$
\gamma_{m}=\frac{E\left[(x-E(x))^{m}\right]}{\sigma^{m}} .
$$

The feature $\gamma_{3}$ is called skewness and the feature $\gamma_{4}$ is called kurtosis. Skewness is related to the asymmetry of the random variable's distribution and it is $\gamma_{3}=0$ if it is symmetrically distributed. If $\gamma_{3}<0$, the data are spread out more to the left of the mean than to the right. The opposite is true if $\gamma_{3}>0$. Kurtosis is a measure of how outlier-prone a distribution is. For a normally distributed random variable we get $\gamma_{4}=3$.

Biased estimators for the sample kurtosis and skewness of $\mathbf{x}^{(z)}$ are given by

$$
\frac{\frac{1}{N} \sum_{n=0}^{N-1}\left(x_{n}^{(z)}-\bar{x}^{(z)}\right)^{m}}{\left(\frac{1}{N} \sum_{n=0}^{N-1}\left(x_{n}^{(z)}-\bar{x}^{(z)}\right)^{2}\right)^{m / 2}}
$$

where

$$
\bar{x}^{(z)}=\frac{1}{N} \sum_{n=1}^{N} x_{n}^{(z)} .
$$

is the average value.

\section{Test rig and measurements}

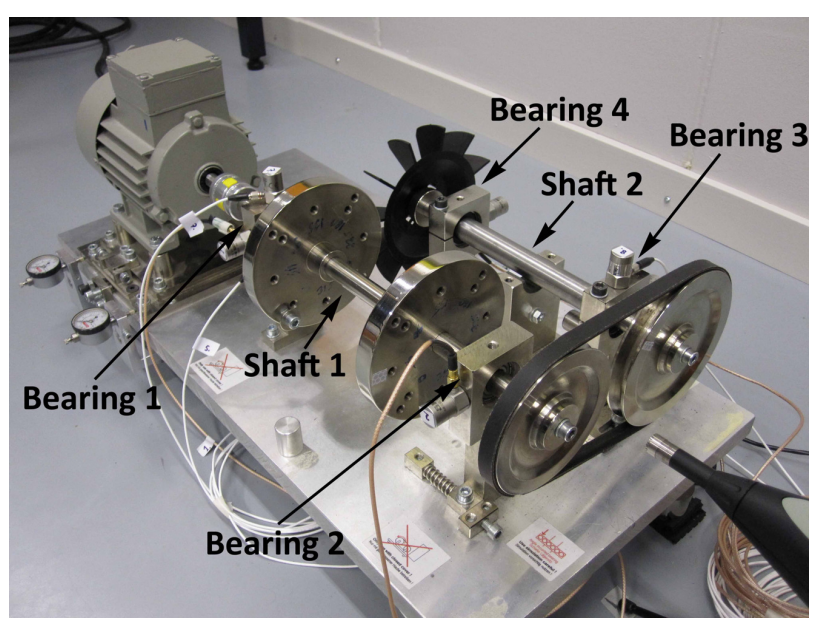

Figure 6. Test rig.

Vibration measurements were recorded from a test rig (figure 6), which consists of a $0.18 \mathrm{~kW}$ AC motor, two shafts supported by two bearings each and a belt transmission between the shafts. A claw clutch with a four-tooth elastic element (spider) couples the motor and the primary shaft. There are also two discs on the primary shaft for attaching unbalance masses, and a small fan is attached to the end of the secondary shaft. More information on the test rig can be found in $[28,29]$.

The measurements were carried out using eight accelerometers, a tachometer and a sound level meter simultaneously. The sound measurements performed using the integrating sound level meter Brüel\&Kjær Type 2239A are not used in this paper, but they have been analysed in [30]. The measurements have also been studied with the help of complex order derivatives and integrals in [31]. The accelerometer types are Wilcoxon Research 726 and IMI 621B51 with linear $( \pm 3 \mathrm{~dB})$ frequency ranges from $2 \mathrm{~Hz}$ to $15 \mathrm{kHz}$ and from $1 \mathrm{~Hz}$ to $20 \mathrm{kHz}$, respectively. The accelerometers were screwed directly into the bearing housing in the radial direction, while a glued mounting pad was used in the axial direction. A horizontal sensor was attached to all of the bearing housings, and vertical and axial sensors were also used on bearings 1 and 3 .

The measurements were performed in the LabVIEW environment by means of three analog input modules (NI 9233) and a four-slot USB chassis 
(NI cDAQ-9174), which ensure simultaneous measurements from all the channels. Each combination of a sensor, cable and measurement channel was calibrated. The sample rate was $50 \mathrm{kHz}$, and 40 to 80 seconds long signals were collected in each case. The tests were carried out with several rotational frequencies between 4 and $23.5 \mathrm{~Hz}$, and various faults and their combinations were implemented. In this paper, only signals with rotational frequency $8 \mathrm{~Hz}$ are utilised in the numerical work. The recorded states of the test rig utilised here are

- initial state

- rotor unbalance mass $5.5 \mathrm{~g}, 11 \mathrm{~g}$ and $16.5 \mathrm{~g}$

- coupling misalignment $0.20 \mathrm{~mm}, 0.35 \mathrm{~mm}$ and 0.50 $\mathrm{mm}$

- rotor unbalance mass $11 \mathrm{~g}$ and coupling misalignment $0.35 \mathrm{~mm}$

- no lubrication in bearing 3

- no lubrication and no cage in bearing 3

- rotor unbalance mass $11 \mathrm{~g}$, coupling misalignment $0.35 \mathrm{~mm}$ and no lubrication, no cage in bearing 3 .

The disk closest to the coupling was used for the unbalance attachment. The unbalances $\left(u_{i}=m_{i} r\right)$ were $276 \mathrm{gmm}, 552 \mathrm{gmm}$ and $828 \mathrm{gmm}$. Coupling misalignment was induced by moving the motor in the horizontal direction while keeping the vertical and angular alignment constant. Two dial gauges were utilised in adjusting the alignment. The cases where unbalance and misalignment occur simultaneously were also recorded before the grease in bearing 3 was washed away. Operating the machine without lubrication in bearing 3 finally caused its cage to crack in several places. The broken cage was removed and the two final states were recorded without it.

\section{Calculations and discussion}

Horizontal measurements from bearing 1 and signal length $N=32768$, i.e. $32768 /(50000 \mathrm{~Hz})=0.65536 \mathrm{~s}$ are utilised in the numerical calculations. The wavelet coefficients are calculated using (20) at scales 1.5, 1.6, $1.7, \ldots 5.9,6.0$. The WTMM ridges are found using the procedure presented in section 2.3 , and the regularities and the corresponding constants A from the fitted lines are calculated with (26). Only ridges for which the constants $\mathrm{A}$ are bigger than or equal to 0.1 are considered.

Figures 7, 8 and 9 show the estimated CWT, the estimated regularities and the corresponding constants A from the acceleration measurements in the case where there are no faults present. Only a handful of local irregularities are present, their constants A are below 0.4 and the regularities are between -0.7 and 0.9. When the misalignment of the claw clutch is $0.20 \mathrm{~mm}$ (figures 10, 11 and 12), the number of local irregularities increases considerably, the Hölder exponents are between -1.4 and 1.2 and the constants A are below 0.6. Figures 13, 14 and 15 show the calculations from the case where the unbalance mass is $5.5 \mathrm{~g}$. The main difference with respect to the case of no faults is that there are roughly twice as many local irregularities in the faulty state.

When the misalignment increases to $0.35 \mathrm{~mm}$, (figures 16, 17 and 18) its effect is clearly already seen in the CWT in the form of periodically repeating ridges. The clearest indicators of this fault are the constants A. We see that we obtain the constants A between 1 and 1.5 once every rotation $(0.125 \mathrm{~s})$, and also halfway between these irregularities there are irregularities in which the constants A are roughly 0.5.

The number of local irregularities increases even more when the misalignment is $0.50 \mathrm{~mm}$ (figures 19, 20 and 21). We now clearly see that the local irregularities occur roughly in bursts that recur four times per revolution of the axis. In one of these bursts, the values of the constants A are between 2.5 and 3 and the other three are always significantly smaller. The Hölder exponents have not changed significantly but are now mainly positive. They also change from negative to positive (and the corresponding constants A decrease) during any singly burst.

The cases of unbalance mass 11 g (figures 22, 23 and 24) and unbalance mass $16.5 \mathrm{~g}$ (figures 25, 26 and 27) are compared on page 13. The number of irregularities increases with bigger unbalance and they occur more clearly as bursts four times per revolution. What separates these faulty states from the misalignment cases are the clearly lower values of constants A (smaller than 0.8).

Figures 28, 29 and 30 show the case of unbalance mass $11 \mathrm{~g}$ together with misalignment $0.35 \mathrm{~mm}$. A higher number of local irregularities is an indication of the two faults occurring simultaneously together, with the bigger values of the constants A occurring twice per revolution.

The cases of a dry bearing (figures 31, 32 and 33) as well as a dry bearing + no cage (figures 34,35 and 36) are characterised by a significant number of local irregularities. The Hölder exponents in the case of a dry bearing apparently fluctuate in quite a random fashion between -3.8 and 1.7, while the constants A reach values up to 3.5 . In the case of no cage + no lubrication, the constants A remain below 0.6 and most of the Hölder exponents are positive, many of them reaching values up to 1.7 .

The case of multiple faults occurring simultaneously (unbalance mass $11 \mathrm{~g}$, misalignment $0.35 \mathrm{~mm}$ and no cage and no lubrication in bearing 3 ) are shown in 
figures 37, 38 and 39. The large number of irregularities and their predominantly positive Hölder exponents clearly reveal the missing cage. At the same time, there are constants A that exceed 1 once in a revolution. They exactly match the effects of $0.35 \mathrm{~mm}$ misalignment. The unbalance does not reveal itself clearly here.

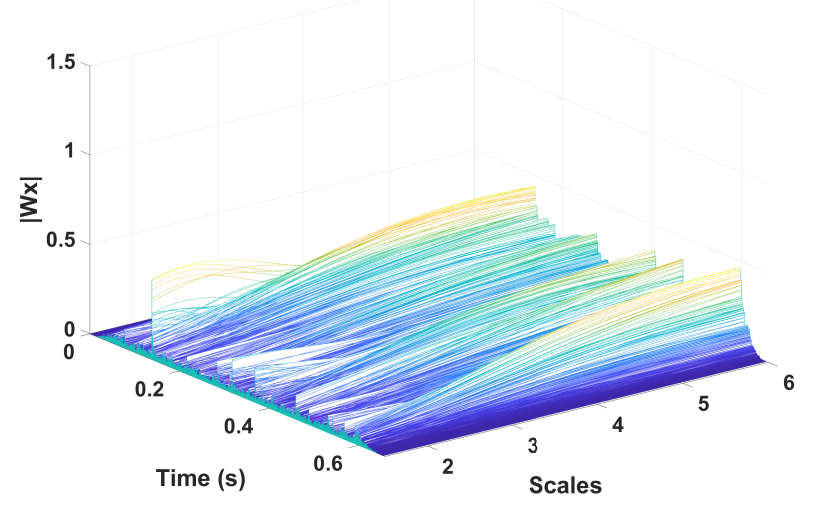

Figure 7. Absolute values of the estimated CWT when no faults are present.

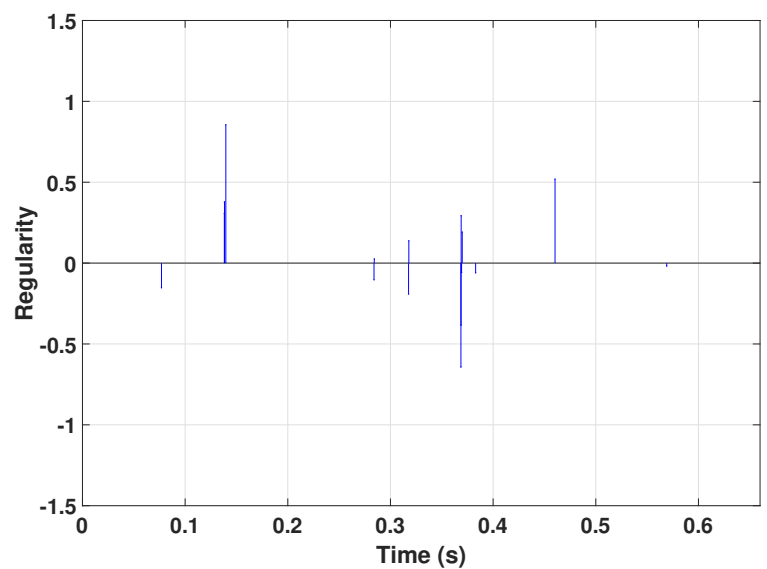

Figure 8. Pointwise Hölder regularities when no faults are present.

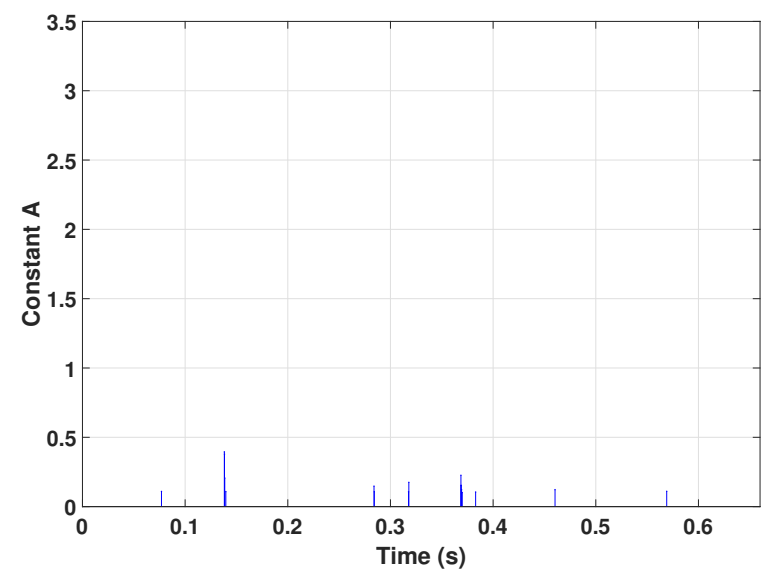

Figure 9. Constants A of the WTMM ridges when no faults are present. 


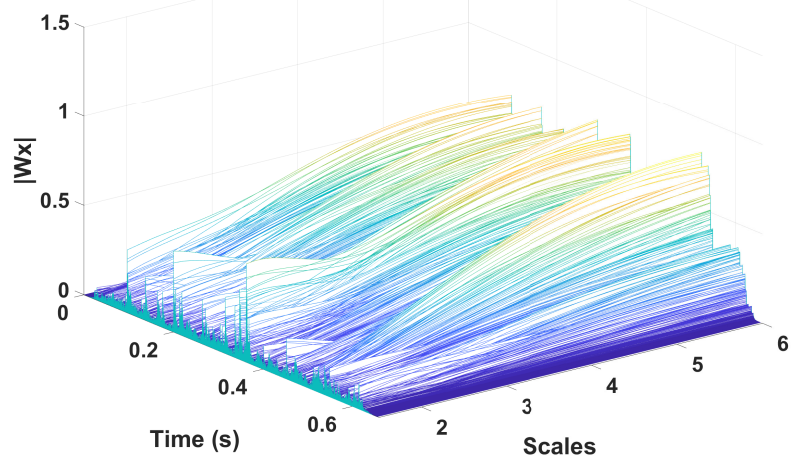

Figure 10. Absolute values of the estimated CWT in the fault case misalignment $0.20 \mathrm{~mm}$.

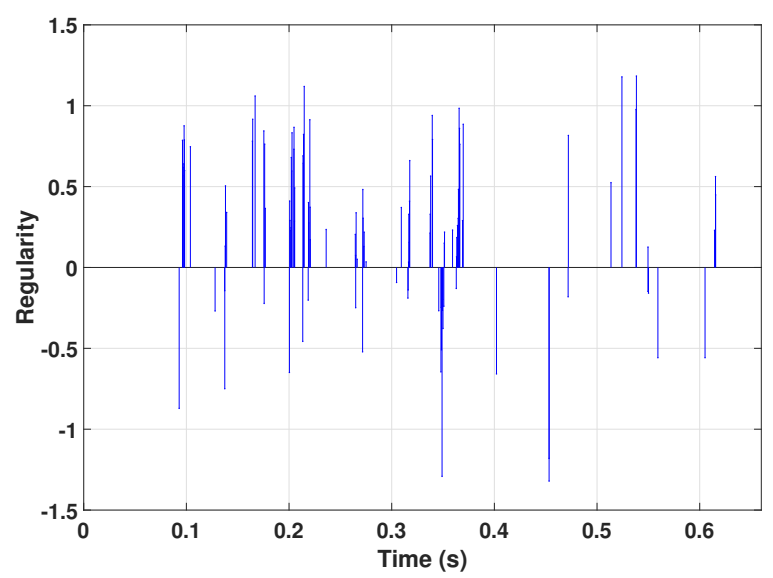

Figure 11. Hölder regularity in the fault case misalignment $0.20 \mathrm{~mm}$.

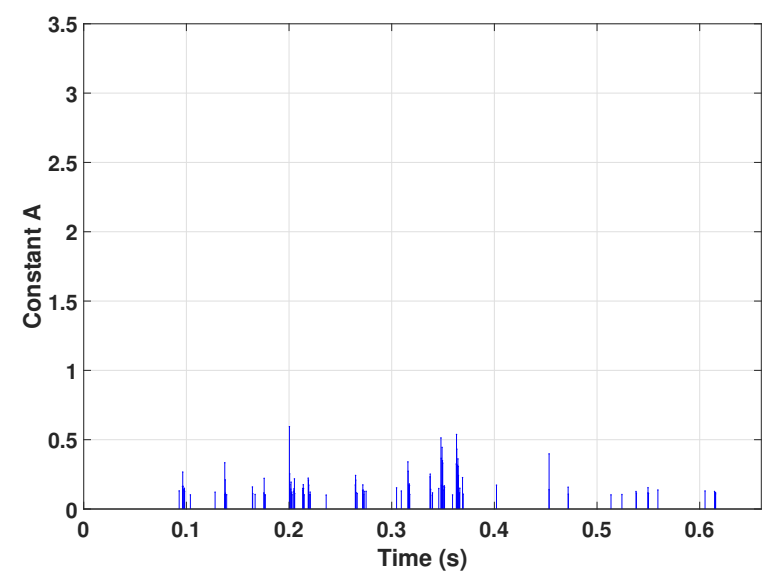

Figure 12. Constants $A$ in the fault case misalignment $0.20 \mathrm{~mm}$.

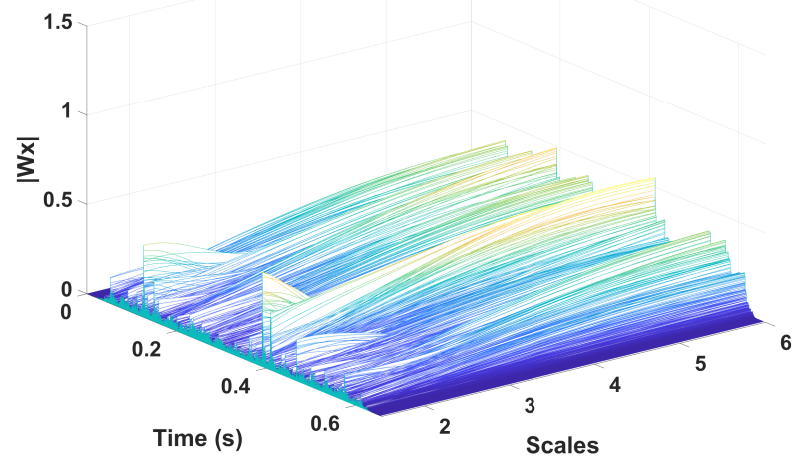

Figure 13. Absolute values of the estimated CWT in the fault case unbalance mass $5.5 \mathrm{~g}$.

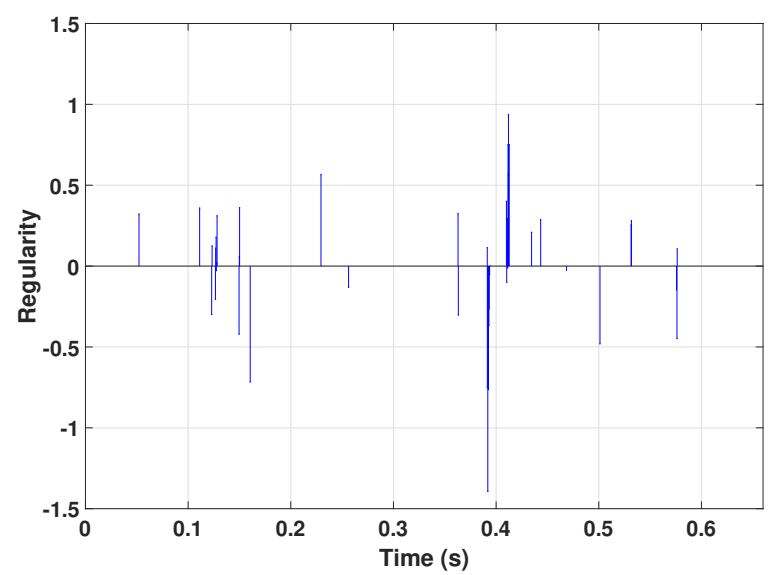

Figure 14. Hölder regularity in the fault case unbalance mass $5.5 \mathrm{~g}$.

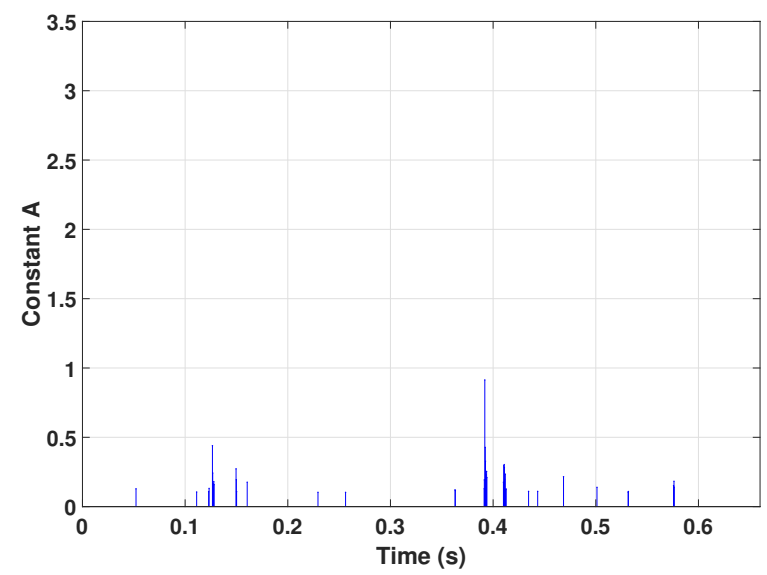

Figure 15. Constants A in the fault case unbalance mass $5.5 \mathrm{~g}$. 


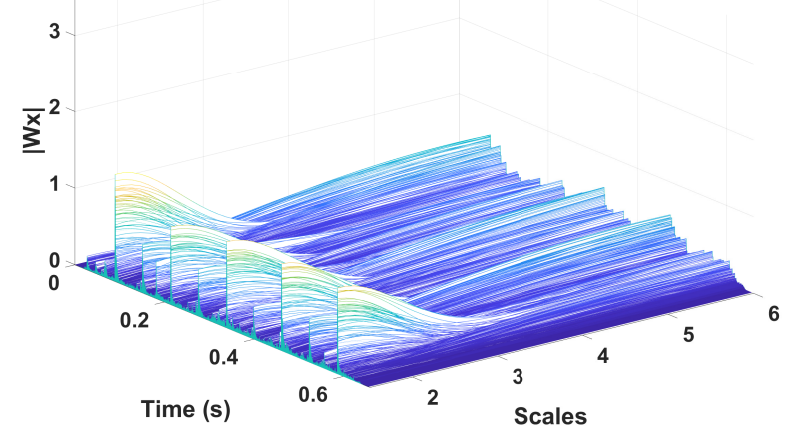

Figure 16. Absolute values of the estimated CWT in the fault case misalignment $0.35 \mathrm{~mm}$.

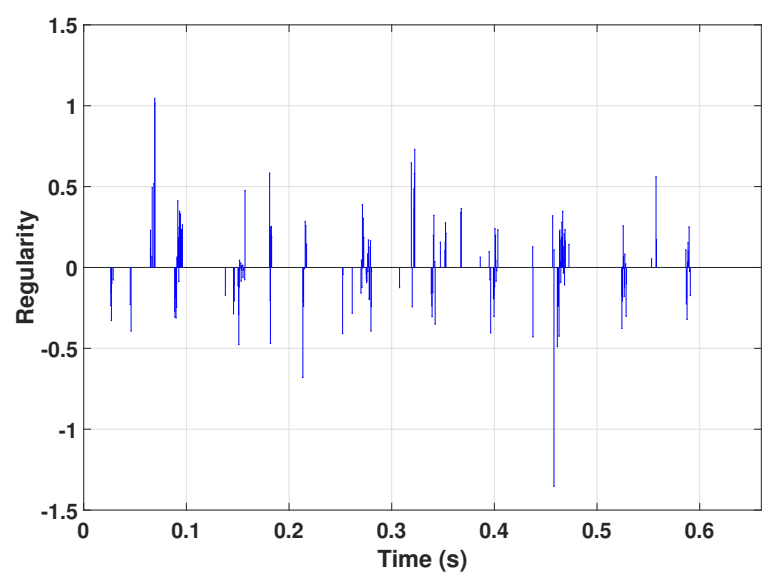

Figure 17. Hölder regularity in the fault case misalignment $0.35 \mathrm{~mm}$.

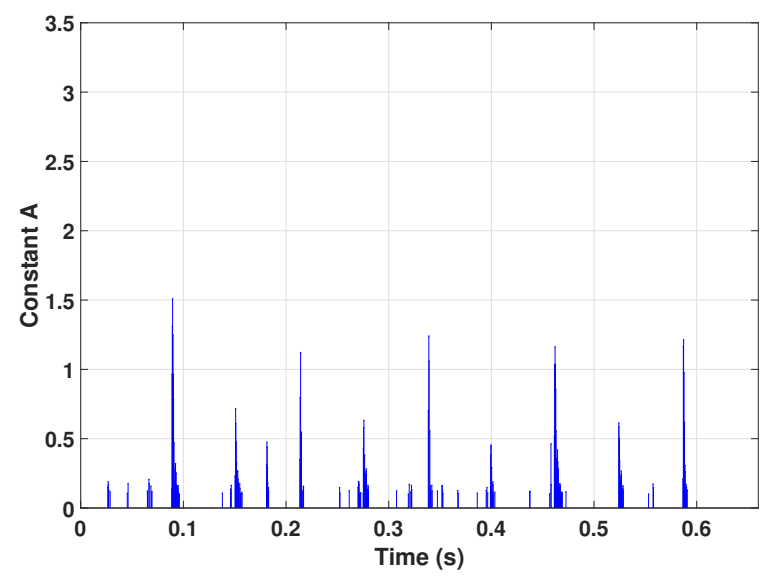

Figure 18. Constants $\mathrm{A}$ in the fault case misalignment $0.35 \mathrm{~mm}$.

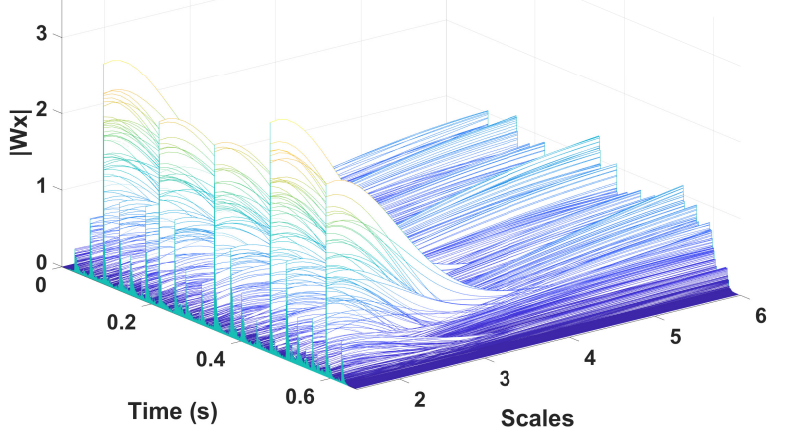

Figure 19. Absolute values of the estimated CWT in the fault case misalignment $0.50 \mathrm{~mm}$.

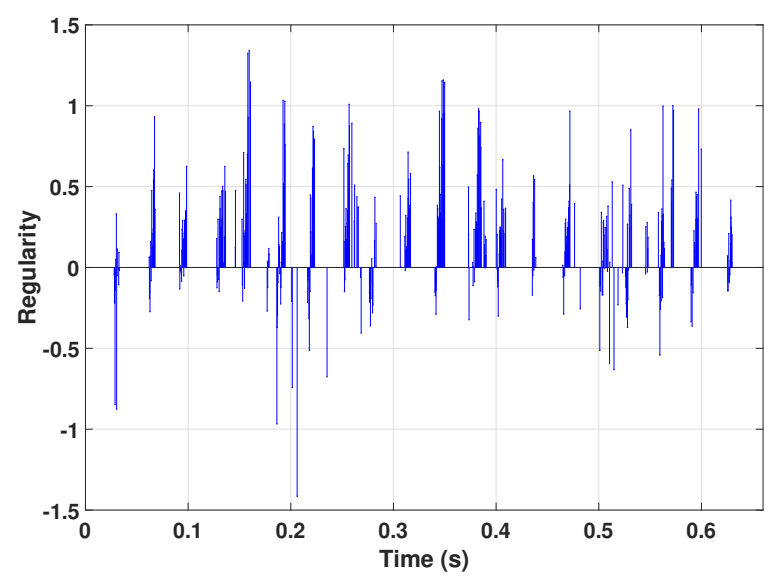

Figure 20. Hölder regularity in the fault case misalignment $0.50 \mathrm{~mm}$.

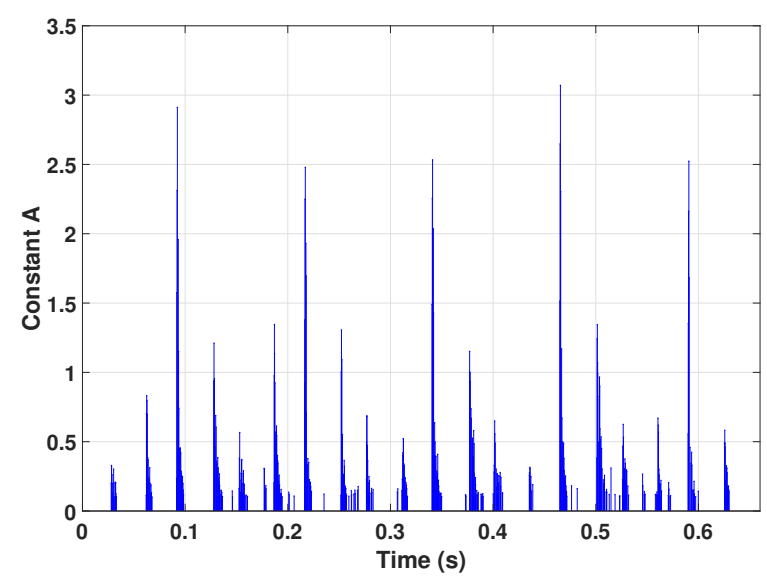

Figure 21. Constants $A$ in the fault case misalignment $0.50 \mathrm{~mm}$. 


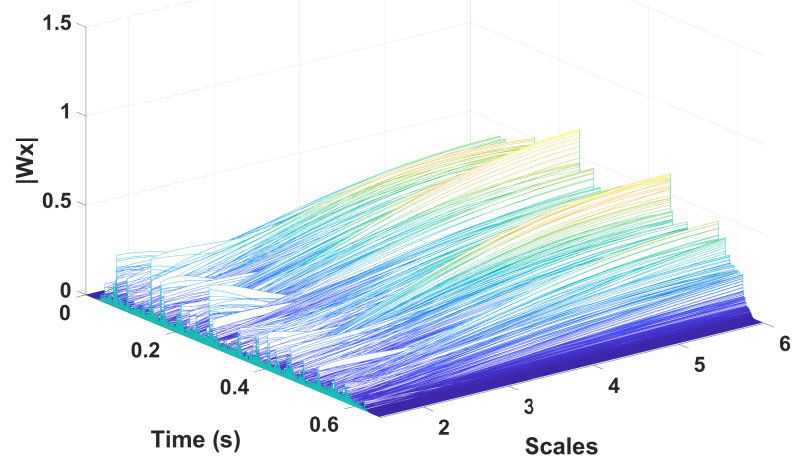

Figure 22. Absolute values of the estimated CWT in the fault case unbalance mass $11.0 \mathrm{~g}$.

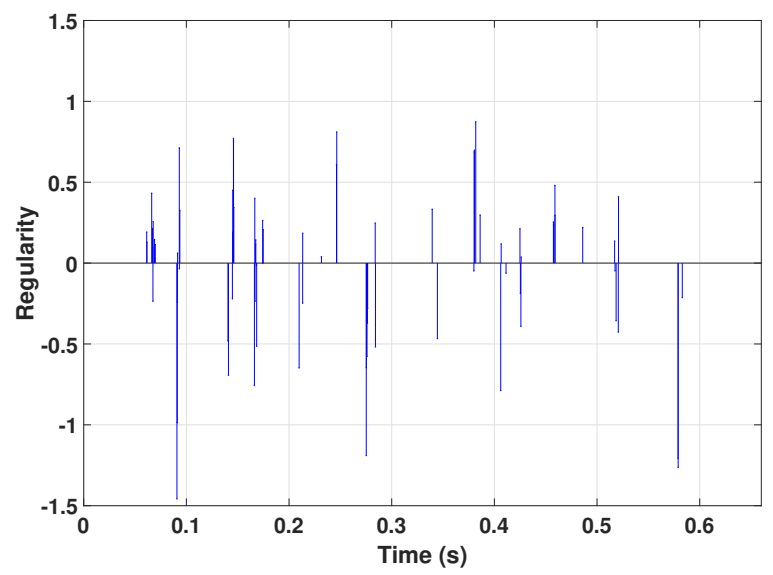

Figure 23. Hölder regularity in the fault case unbalance mass $11.0 \mathrm{~g}$.

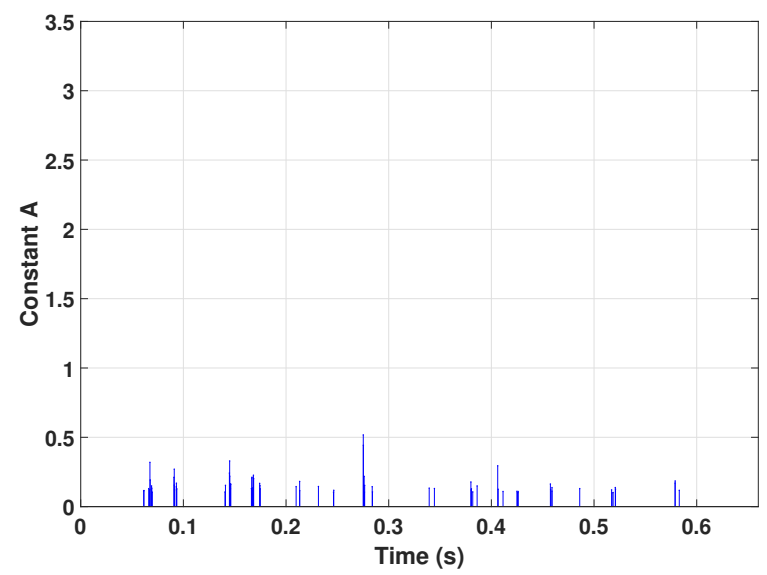

Figure 24. Constants $\mathrm{A}$ in the fault case unbalance mass $11.0 \mathrm{~g}$.

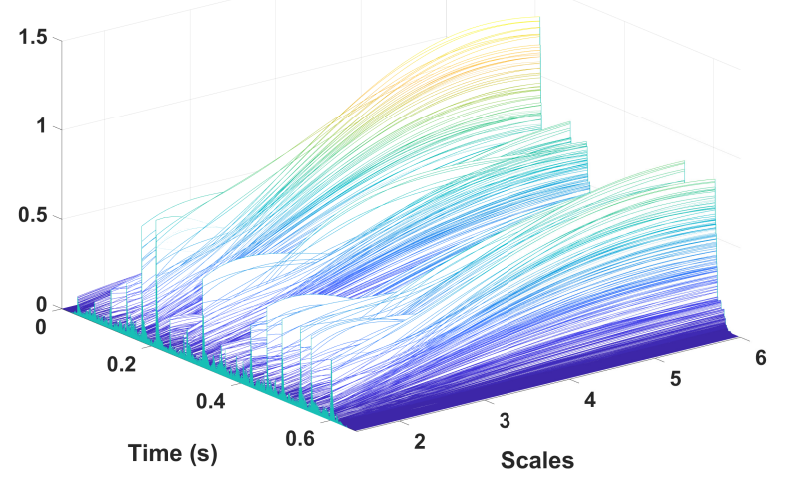

Figure 25. Absolute values of the estimated CWT in the fault case unbalance mass $16.5 \mathrm{~g}$.

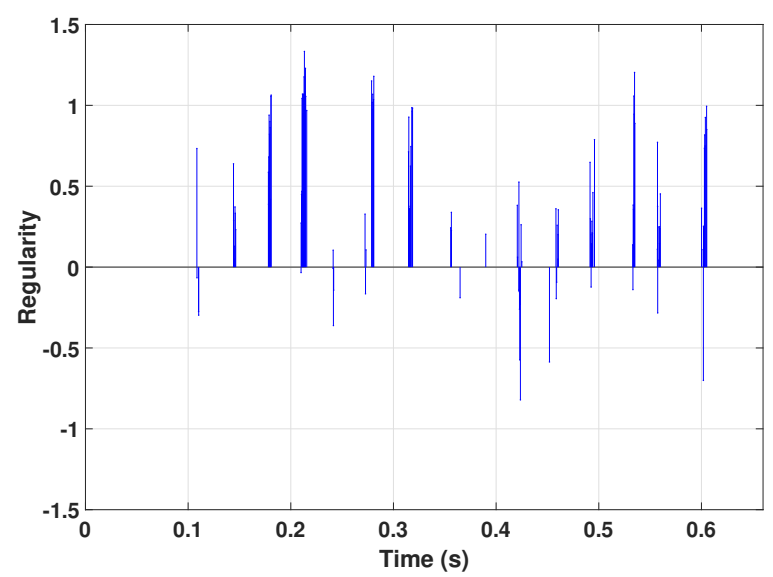

Figure 26. Hölder regularity in the fault case unbalance mass $16.5 \mathrm{~g}$.

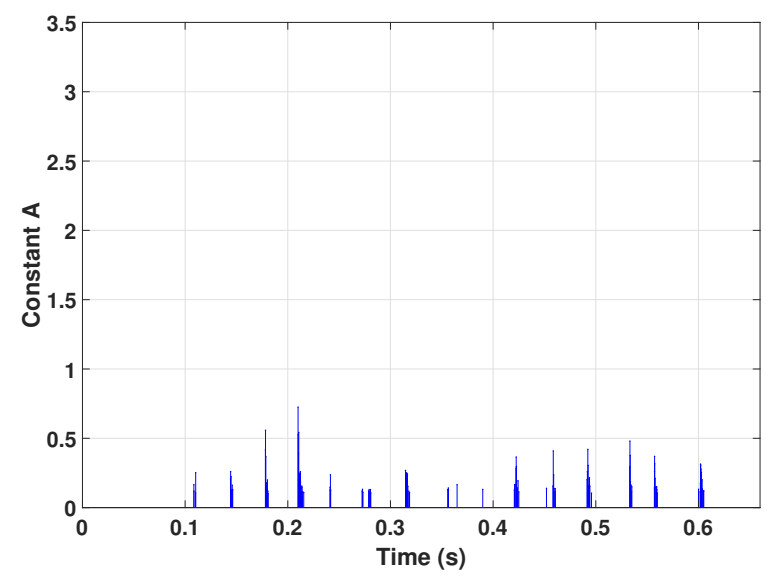

Figure 27. Constants $A$ in the fault case unbalance mass $16.5 \mathrm{~g}$. 


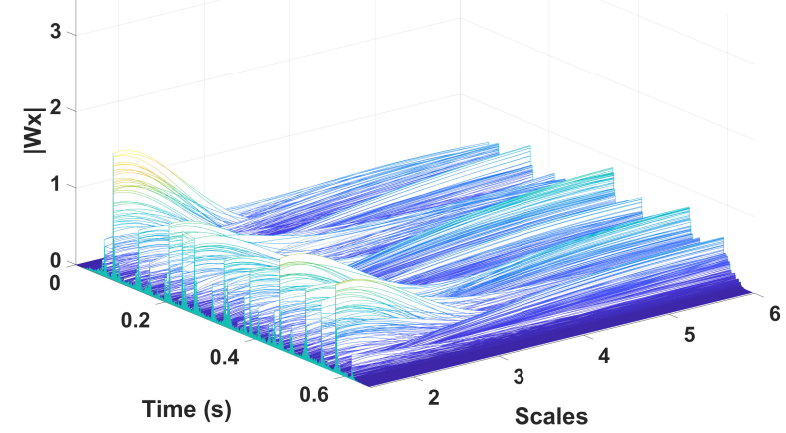

Figure 28. Absolute values of the estimated CWT in the fault case unbalance mass $11.0 \mathrm{~g}$ and misalignment $0.35 \mathrm{~mm}$.

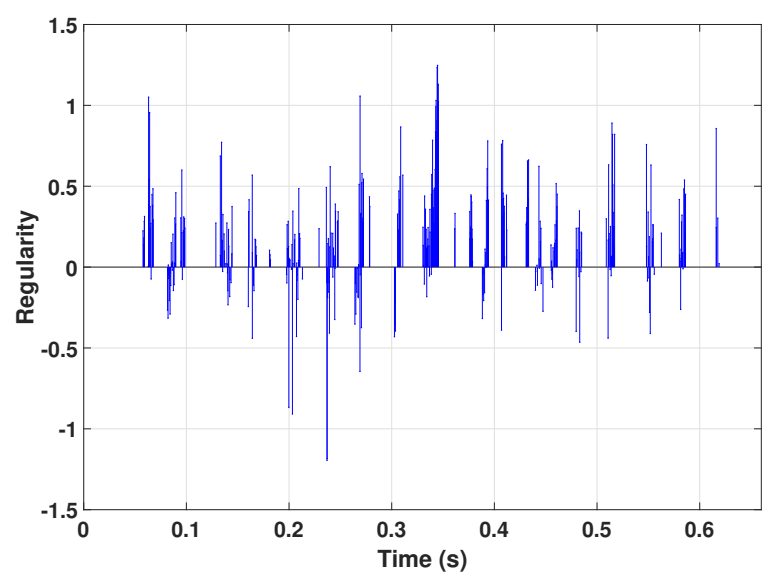

Figure 29. Hölder regularity in the fault case unbalance mass $11.0 \mathrm{~g}$ and misalignment $0.35 \mathrm{~mm}$.

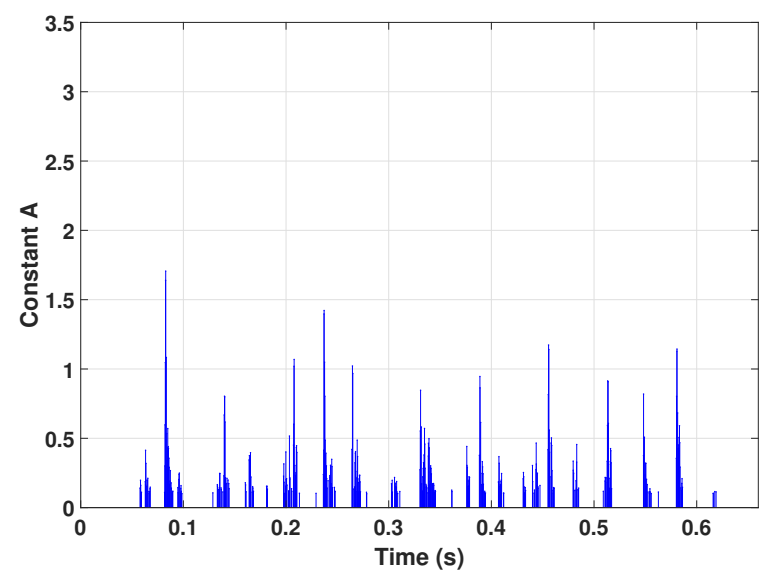

Figure 30. Constants $\mathrm{A}$ in the fault case unbalance mass $11.0 \mathrm{~g}$ and misalignment $0.35 \mathrm{~mm}$.

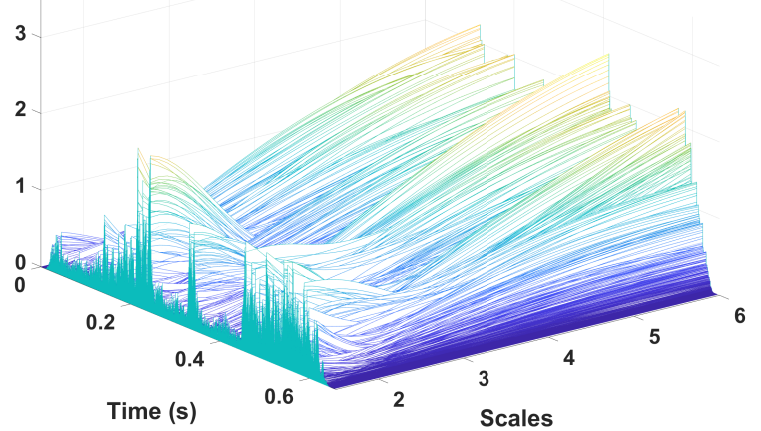

Figure 31. Absolute values of the estimated CWT in the fault case no lubrication in bearing 3 .

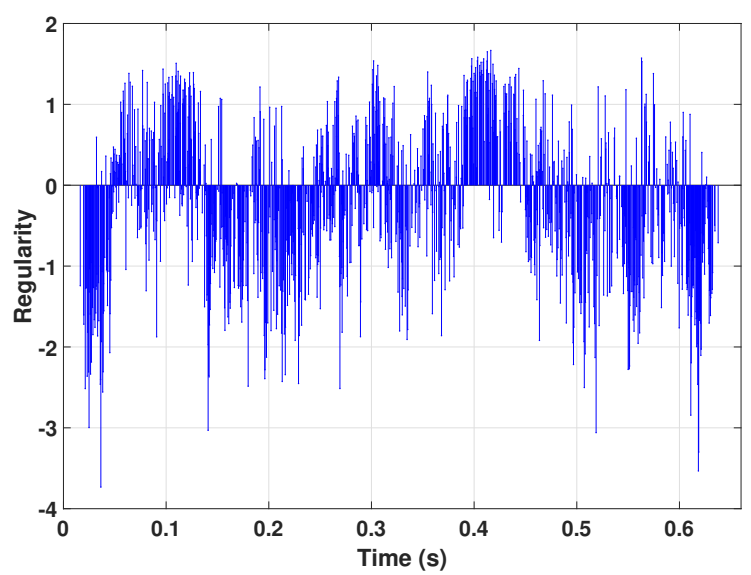

Figure 32. Hölder regularity in the fault case no lubrication in bearing 3 .

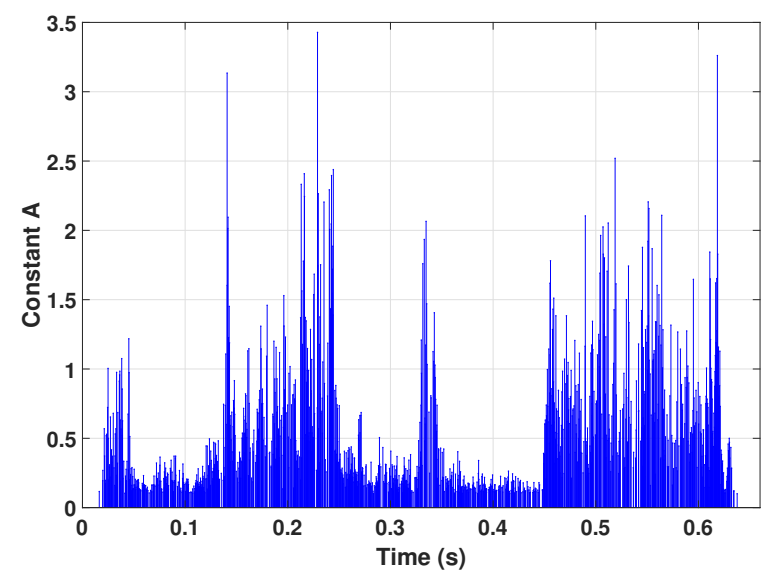

Figure 33. Constants $\mathrm{A}$ in the fault case no lubrication in bearing 3 . 


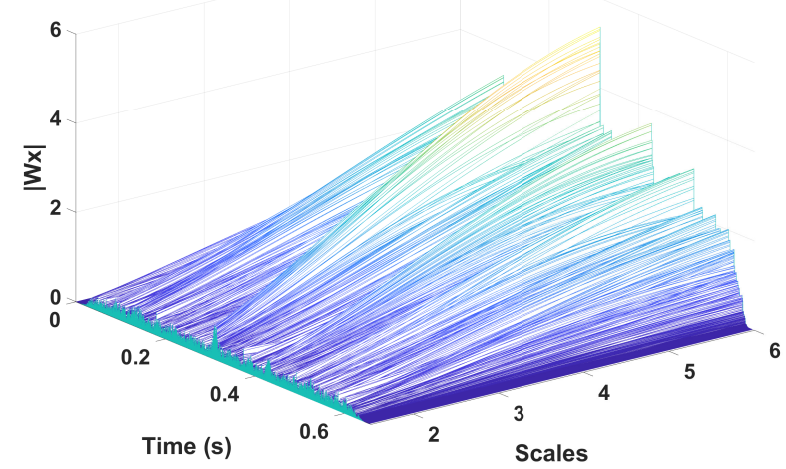

Figure 34. Absolute values of the estimated CWT in the fault case no cage and no lubrication in bearing 3 .

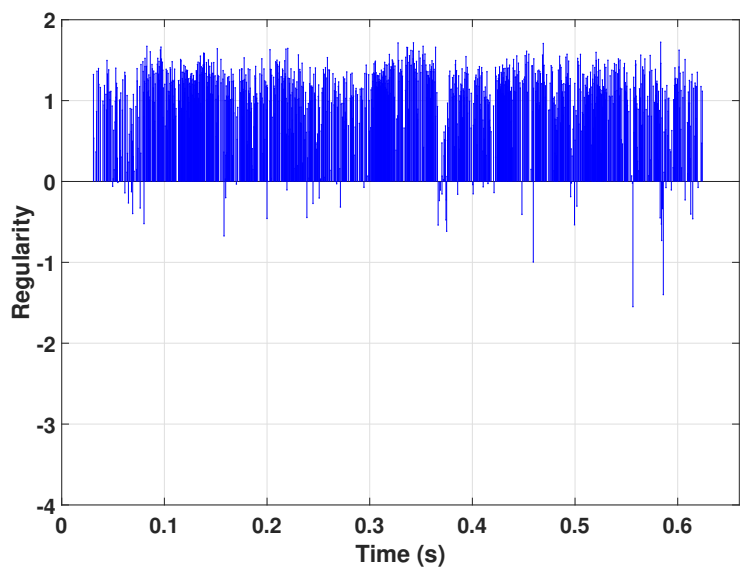

Figure 35. Hölder regularity in the fault case no cage and no lubrication in bearing 3 .

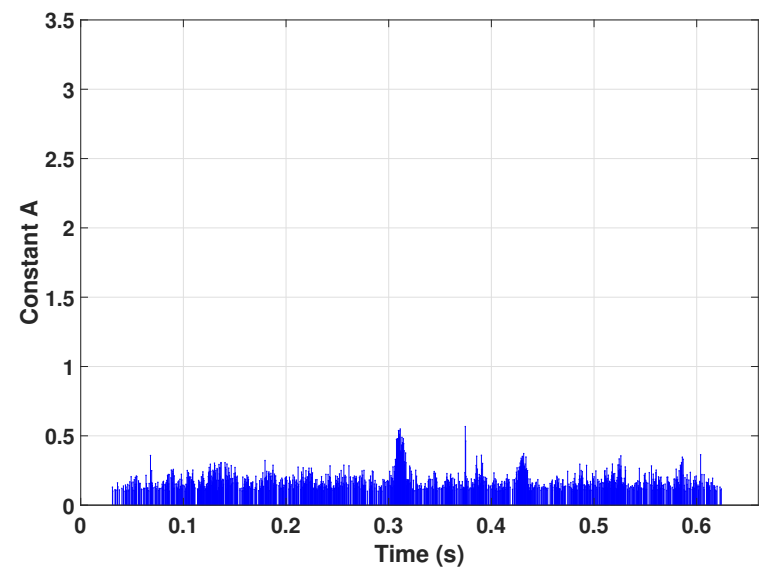

Figure 36. Constants $\mathrm{A}$ in the fault case no cage and no lubrication in bearing 3 .

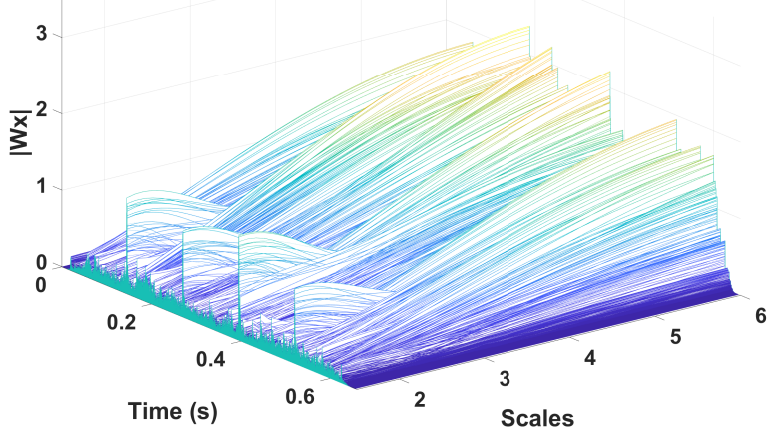

Figure 37. Absolute values of the estimated CWT in the fault case unbalance mass $11 \mathrm{~g}$, misalignment $0.35 \mathrm{~mm}$ and no cage and no lubrication in bearing 3 .

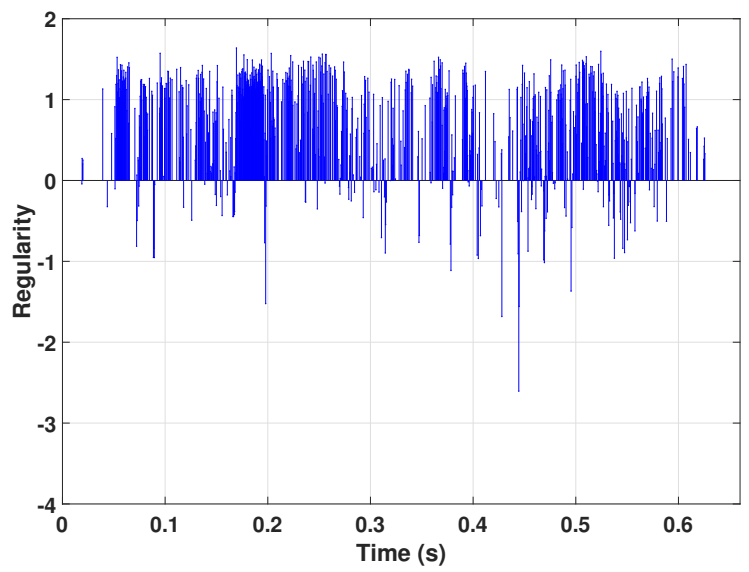

Figure 38. Hölder regularity in the fault case unbalance mass $11 \mathrm{~g}$, misalignment $0.35 \mathrm{~mm}$ and no cage and no lubrication in bearing 3 .

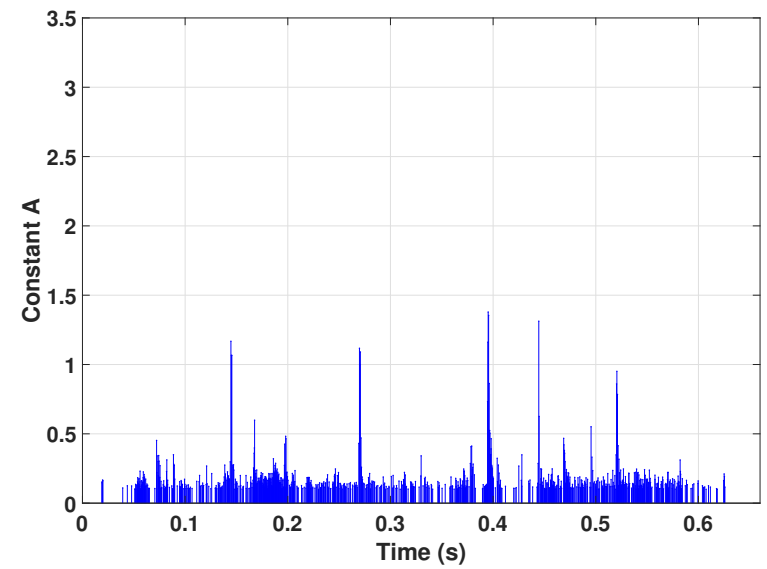

Figure 39. Constants $A$ in the fault case unbalance mass $11 \mathrm{~g}$, misalignment $0.35 \mathrm{~mm}$ and no cage and no lubrication in bearing 3. 
Based on the analysis done so far, we can postulate that the number of pointwise irregularities and their Hölder exponents can be used to detect misalignment and bearing faults. However, effectively diagnosing unbalance seems to be impossible using these methods alone. To verify these findings, a bigger set of data was analysed. For the fault cases no cage + no lubrication in bearing 3 and the misalignment 0.35 $\mathrm{mm}+$ unbalance $11.0 \mathrm{~g}+$ no cage + no lubrication in bearing 3, a total of 65 segments of length $N=32768$ were analysed and for all the other fault states 122 segments were analysed. The lengths of the original vibration recordings dictated the number of segments. As a result, the total number of analysed segments was 1228.

For all the segments, exactly the same signal processing methods were used as before to locate the pointwise irregularities and to estimate the Hölder exponents and the constants A. Several different features were then tested, of which the most simple and promising seemed to be the number of irregularities (i.e. the number of ridges in the CWT whose constant A is above 0.1) and the mean Hölder exponent of all the irregularities. Based on previous research [30], it was known that unbalance can be detected from low order norms of integrated acceleration measurements and that misalignment and bearing faults are more sensitive to higher order norms of differentiated acceleration measurements. For this reason, we also computed the values $\left\|x^{(1)}\right\|_{1,1 / N}, \quad\left\|x^{(3)}\right\|_{2,1 / N}$ and $\left\|x^{(3)}\right\|_{8,1 / N}$ for every segment. The values $\left\|x^{(1)}\right\|_{1,1 / N}$ complement the local regularity analysis and the performance of values $\left\|x^{(3)}\right\|_{2,1 / N}$ and $\left\|x^{(3)}\right\|_{8,1 / N}$ are compared against the local regularity analysis. The integrated values were also filtered with an ideal filter to the range $5-25000 \mathrm{~Hz}$ and the differentiated values to the range $5-10000 \mathrm{~Hz}$. We calculated also kurtosis and skewness of $x^{(z)}$ for $z=0,1,2,3,4$. Of these, only kurtosis of $x^{(3)}$ performed moderately well and is thus the only feature to be included in the further analysis.

Figure 40 shows $\left\|x^{(3)}\right\|_{8,1 / N}$ vs. $\left\|x^{(1)}\right\|_{1,1 / N}$. We see that unbalance is quite well identified using velocity signals and that only the smallest unbalance overlaps the no fault state. The jerk signals adequately separate the misalignment states, although here the smallest misalignment state also overlaps with the no fault state and the larger misalignment states. The no lubrication state is clearly identified, though its variance in the $\left\|x^{(3)}\right\|_{8,1 / N}$ values is quite large. The cases of multiple faults occurring simultaneously severely overlap with the no cage in bearing 3 state. Overall, the results can be considered adequate, but not outstanding. Figure 41 shows $\left\|x^{(3)}\right\|_{2,1 / N}$ vs. $\left\|x^{(1)}\right\|_{1,1 / N}$ and it seems that the rms of jerk signals separates the cases of multiple faults occurring simultaneously much better than $\left\|x^{(3)}\right\|_{8,1 / N}$ does. The order of the norm can play an important role when using traditional features. Figure 42 shows kurtosis of $x^{(3)}$ vs. $\left\|x^{(1)}\right\|_{1,1 / N}$. There is a lot of variance in the kurtosis values and the different fault states overlap a lot. All the kurtosis values are greater than or equal to 3 , which is the kurtosis of a Gaussian distribution. Interestingly, all the values from the fault state no cage + dry bearing are between 3 and 4 and all the values from the fault state $0.35 \mathrm{~mm}$ misalignment + no cage + dry bearing are just slightly bigger.

Figure 43 shows the number of local irregularities vs. $\left\|x^{(1)}\right\|_{1,1 / N}$. Here, the misalignment states are much better separated. The cases of multiple faults occurring simultaneously are clearly separated and all the three states that include some bearing faults have over 600 local irregularities. The cases of no lubrication in bearing 3 and no cage + no lubrication in bearing 3 overlap to some extent.

Figure 44 shows the number of local irregularities vs. their mean Hölder regularities. We see that this can complement the data shown in figure 43 , since the mean Hölder regularity perfectly separates the cases of no lubrication in bearing 3 and no cage + no lubrication in bearing 3 . The regularities are between -1.4 and 0.3 in the case of no lubrication in bearing 3, between 0.9 and 1.2 in the case of no cage + no lubrication in bearing 3 and between 0.6 and 1 in the case of misalignment $0.35 \mathrm{~mm}+$ unbalance $11.0 \mathrm{~g}+$ no cage + no lubrication in bearing 3. The regularities range between -0.1 and 0.4 in the fault case misalignment $0.35 \mathrm{~mm}+$ unbalance $11.0 \mathrm{~g}$, between 0 and 0.3 in the fault case $0.35 \mathrm{~mm}$ misalignment and between 0.2 and 0.4 in the fault case $0.50 \mathrm{~mm}$ misalignment. All the other fault states have mean Hölder regularities between 0.4 and 0.8 and they significantly overlap in figure 44.

Quadratic classifiers were tested on the data sets of figures 40,41, 42, 43 and 44 in order to obtain some numerical validation for our observations. A quadratic classifier is constructed by simply computing the sample mean and covariance of each class (different faults states in this case) and using them to fit 2D multinormal distributions to represent the classconditional probability distributions. A new vector is assigned to the class whose class-conditional density has the maximum value at that point (in this case, we assume that all the classes are equally likely to appear, so they all have equal prior probabilities). This is referred to as Quadratic Discriminant Analysis (QDA) in [32]. To accurately estimate the error of the classifiers, we use the leave-one-out validation method. This means that one data vector is left out, the classifier is constructed without it and then tested with it, and the data of erroneous or correct classification are 


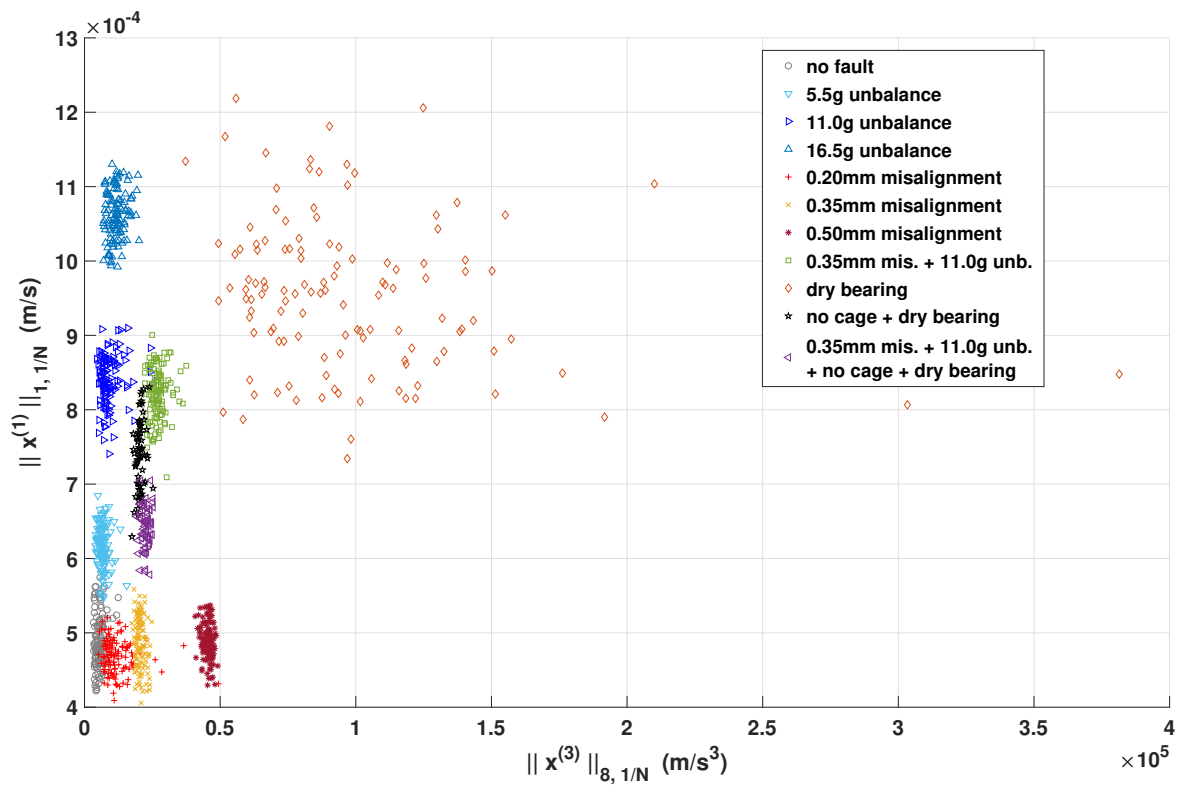

Figure 40. Generalised norms of order 8 of jerk signals and arithmetic means of absolute values of velocity signals.

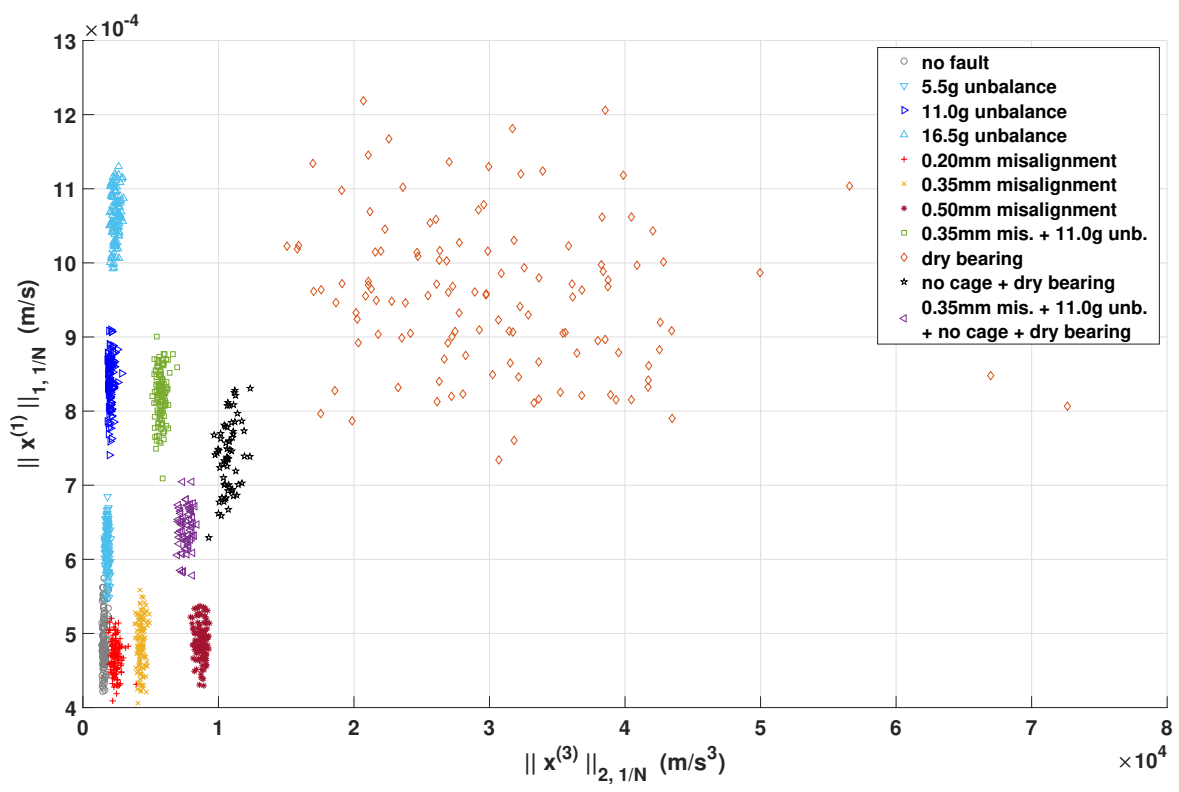

Figure 41. Root mean square values of jerk signals and arithmetic means of absolute values of velocity signals.

recorded. The procedure is repeated for all the 1228 data vectors. Since only one data vector is always left out, the classifiers in the different steps of calculation are nearly identical but the error estimates are not optimistically biased since we have separate training and testing data.

The number of wrong classifications in each class and their sums are collected in table 1 for the five classifiers based on figures $40,41,42,43$ and 44 . This verifies our earlier observations, namely that the data sets in figures 43 and 41 perform overall very well, the data in figure 40 perform overall adequately and the data in figure 44 perform without errors when bearing problems are present but very poorly in almost all of the other situations. The performance of the data in figure 42 is the worst.

The estimated classification errors of these QDA classifiers are calculated in table 2. A combined classifier is also included in the table. This classifier first makes a binary decision to use the data in figure 


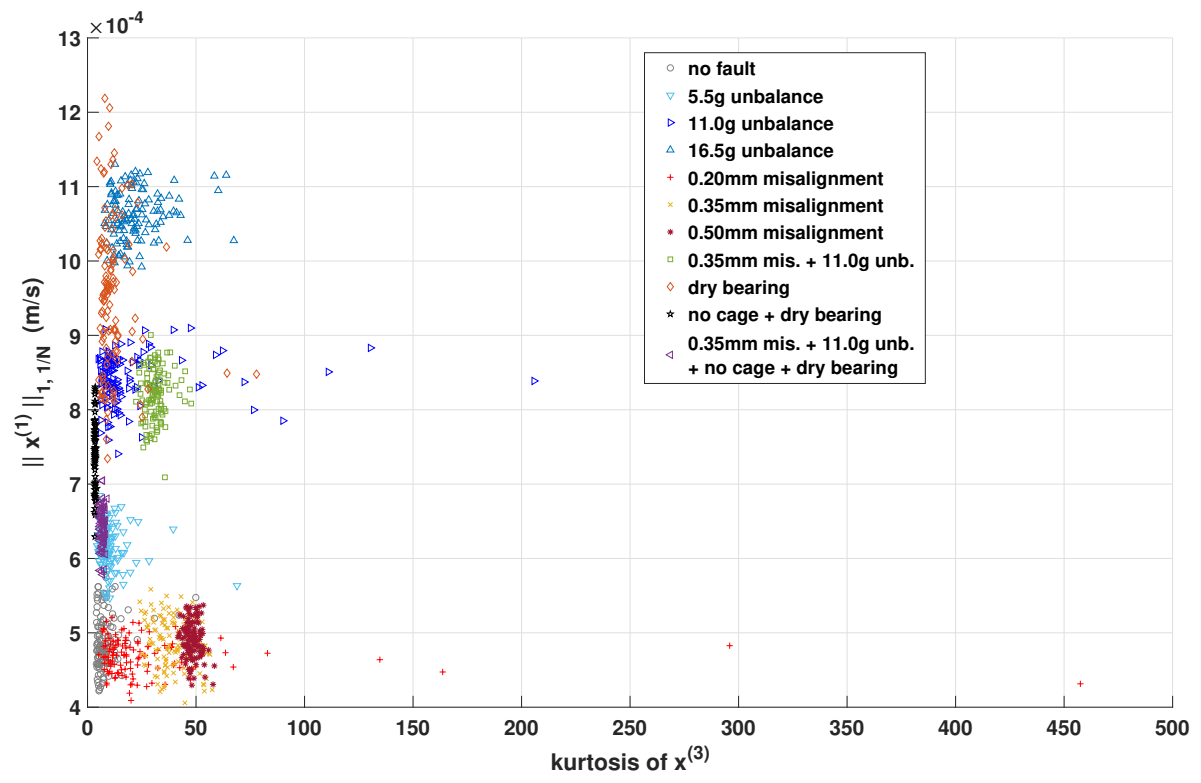

Figure 42. Kurtosis of jerk signals and arithmetic means of absolute values of velocity signals.

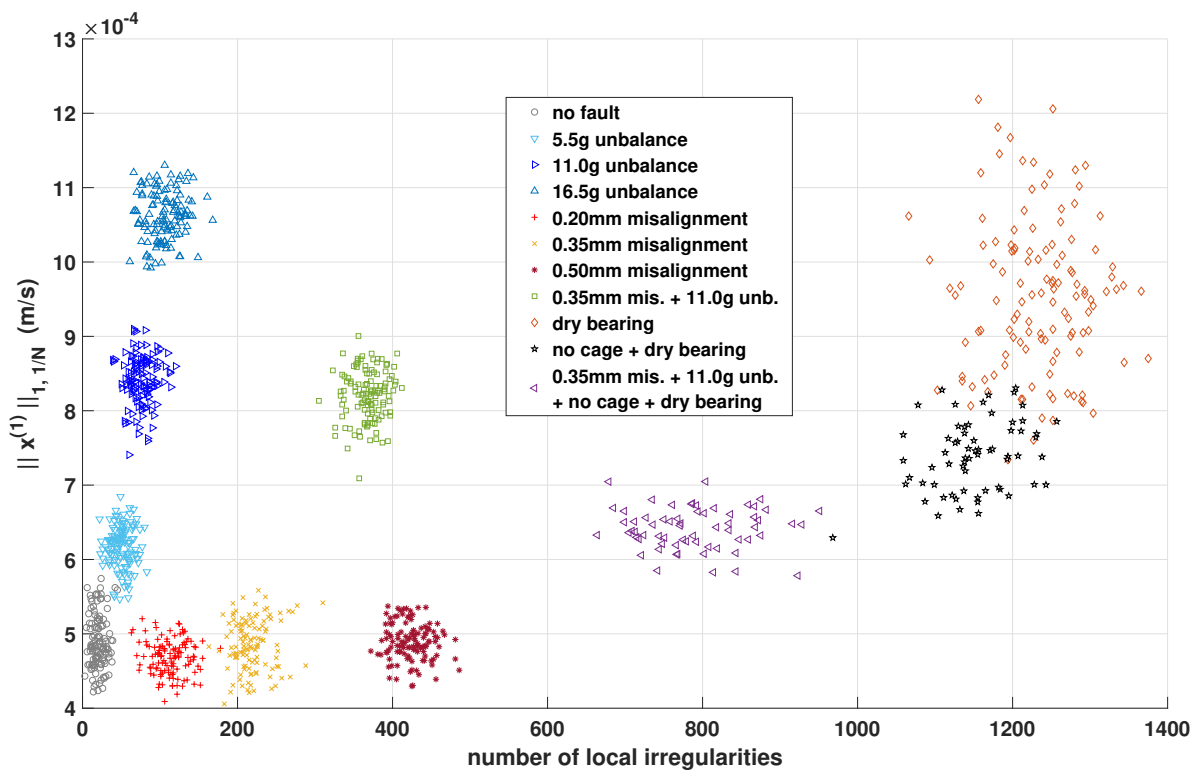

Figure 43. The number of local irregularities and arithmetic means of absolute values of velocity signals.

43 if the number of local irregularities is below 600 and the data in figure 44 if the number is 600 or more. In this way, we can achieve a classification error of as little as $0.3 \%$, which is just 4 misclassifications out of 1228 data vectors. Two of these misclassifications were from the class of no faults and one from the class $0.2 \mathrm{~mm}$ misalignment and one from the class $0.35 \mathrm{~mm}$ misalignment. Using only the number of local irregularities and the values $\left\|x^{(1)}\right\|_{1,1 / N}$ also gives a relatively good classification error of $1.3 \%$ and using $\left\|x^{(3)}\right\|_{2,1 / N}$ and $\left\|x^{(1)}\right\|_{1,1 / N}$ gives a classification error of only $0.8 \%$. With the values $\left\|x^{(3)}\right\|_{8,1 / N}$ and $\left\|x^{(1)}\right\|_{1,1 / N}$, we obtain a significantly worse classification error of $4.7 \%$.

Although the decision boundaries in a QDA classifier are second degree curves, the classes are so well separated visually in figure 43 that we can also pick simple thresholds for the purpose of a very quick approximate fault diagnostics. First we suppose 


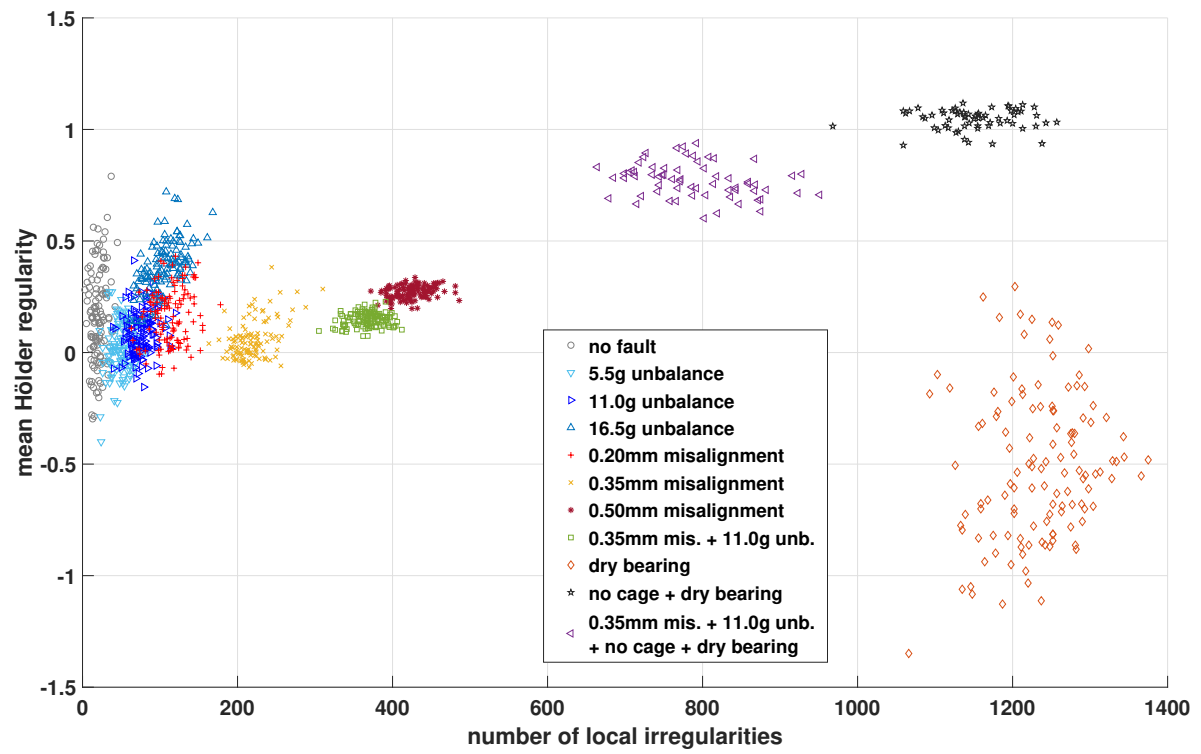

Figure 44. The number of local irregularities and their mean Hölder exponents.

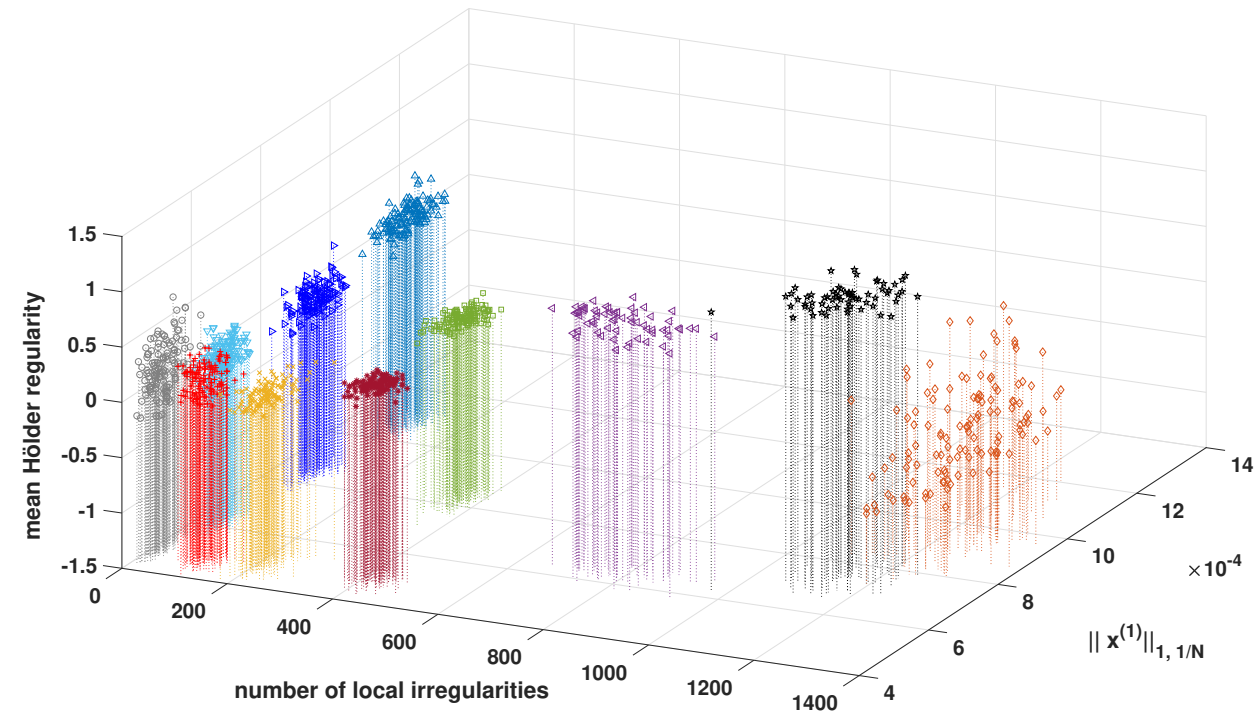

Figure 45. The number of local irregularities, their mean Hölder exponents and arithmetic means of absolute values of velocity signals.

that there are fewer than 600 local irregularities, and thus bearing problems are not likely. Now unbalance $5.5 \mathrm{~g}$ likely occurs when $\left\|x^{(1)}\right\|_{1,1 / N}$ is between 0.55 and $0.7 \mathrm{~mm} / \mathrm{s}$. When $\left\|x^{(1)}\right\|_{1,1 / N}$ is between 0.7 and $0.95 \mathrm{~mm} / \mathrm{s}$, we have an unbalance of $11.0 \mathrm{~g}$ (and combined with $0.35 \mathrm{~mm}$ misalignment if the number of local irregularities is between 300 and 500). Finally, the fault state unbalance $16.5 \mathrm{~g}$ occurs when $\left\|x^{(1)}\right\|_{1,1 / N}$ is bigger than $0.95 \mathrm{~mm} / \mathrm{s}$. Misalignments of $0.20 \mathrm{~mm}, 0.35 \mathrm{~mm}$, and $0.50 \mathrm{~mm}$ occur when the number of irregularities is between 50 and 170, between 170 and 350, and between 350 and 500 respectively. The combined fault state misalignment $0.35 \mathrm{~mm}+$ unbalance $11.0 \mathrm{~g}+$ no cage + no lubrication in bearing 3 occurs when the number of irregularities is between 650 and 960 and $\left\|x^{(1)}\right\|_{1,1 / N}$ is between 0.57 and 0.71 $\mathrm{mm} / \mathrm{s}$. Finally, when the number of irregularities is over 960 , we have either a dry bearing or no cage + 
Table 1. Number of wrong classifications in each class and their sum when using the data from figures $40,41,42,43$ and 44 , a quadratic classifier and leave-one-out cross-validation.

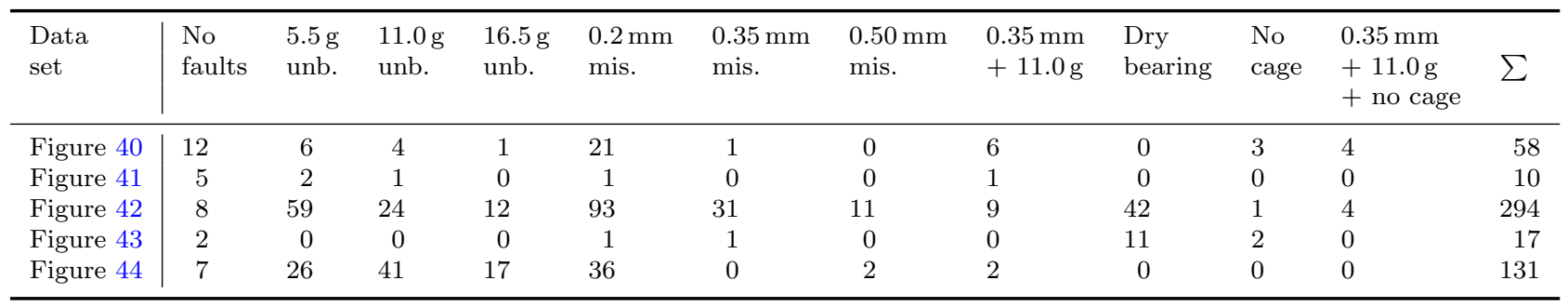

Table 2. Estimated classification errors for the three quadratic classifiers based on the data from figures 40, 41, 42 and 43 and 44 plus a combined classifier using the data from figure 43 when the amount of irregularities is under 600, and otherwise based on the data from figure 44 .

\begin{tabular}{lr}
\hline Data set & Estimated classification error \\
\hline Figure 40 & $58 / 1228 \approx 4.7 \%$ \\
Figure 41 & $10 / 1228 \approx 0.8 \%$ \\
Figure 42 & $294 / 1228 \approx 23.9 \%$ \\
Figure 43 & $17 / 1228 \approx 1.3 \%$ \\
Figure 44 & $131 / 1228 \approx 10.7 \%$ \\
Figures 43 and 44 combined & $4 / 1228 \approx 0.3 \%$ \\
\hline
\end{tabular}

dry bearing. The fault case dry bearing alone is more likely if $\left\|x^{(1)}\right\|_{1,1 / N}$ is over $0.8 \mathrm{~mm} / \mathrm{s}$, but a better way is to use the mean Hölder regularity from figure 44 and decide that the case of a dry bearing alone occurs when the mean Hölder regularity is below 0.5 . We have visualised these three features also in a single three dimensional figure 45 . The colors and markers for the different fault states are the same as in the previous figures.

\section{Conclusions}

The local regularity analysis of vibration signals has a lot of potential for condition monitoring and fault diagnostics purposes. In this paper, we have seen that in a small test rig a coupling misalignment of a claw clutch causes irregularities in the acceleration signals that are very evenly spaced (once or twice in a cycle) and have both positive and negative Hölder exponents (mostly positive when the misalignment is more severe). On the other hand, missing lubrication in a bearing causes unevenly spaced irregularities that mostly have negative Hölder exponents. Removing the cage from the poorly lubricated bearing made its vibrations more regular, but the number of irregularities was still huge. These bearing faults represent quite severe situations in real machines.

Unbalance is best observed not from the acceleration signal itself but from its integrals. Unbalance caused the test rig to oscillate mainly at the shaft frequency. This can even be observed when bearing problems and misalignment occur simultaneously. In this state of simultaneous faults, the misalignment and bearing faults are still clearly observed from the acceleration measurements. By combining just three features (the arithmetic mean of absolute values of velocity signals, the number of local irregularities and their mean Hölder regularity) a simple quadratic classifier was constructed that separated all the fault states with an estimated classification error of just $0.3 \%$.

\section{Acknowledgments}

For the first half of the year 2018, the first author's research has been funded by the Finnish Cultural Foundation, North Ostrobothnia Regional Fund. The first author also wishes to thank the Auramo-Foundation, Riitta and Jorma J. Takanen Foundation and Walter Ahlström Foundation for their support for his doctoral studies.

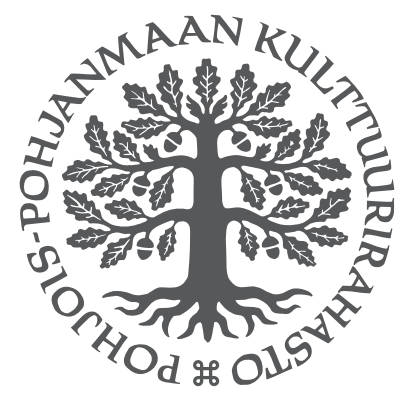

\section{ORCID iDs}

Juhani Nissilä:

https://orcid.org/0000-0001-6908-0406

Jouni Laurila:

https://orcid.org/0000-0001-6397-6180 


\section{References}

[1] Yan X, Jia M and Xiang L 2016 Compound fault diagnosis of rotating machinery based on OVMD and a 1.5dimension envelope spectrum Meas. Sci. Technol. 27 075002

https://doi.org/10.1088/0957-0233/27/7/075002

[2] Zhang D, Yu D and Zhang W 2015 Energy operator demodulating of optimal resonance components for the compound faults diagnosis of gearboxes Meas. Sci. Technol. 26115003 https://doi.org/10.1088/0957-0233/26/11/115003

[3] Qiao Z and Pan Z 2015 SVD principle analysis and fault diagnosis for bearings based on the correlation coefficient Meas. Sci. Technol. 26085014 https://doi.org/10.1088/0957-0233/26/8/085014

[4] Lahdelma S and Juuso E 2011 Signal processing and feature extraction by using real order derivatives and generalised norms. Part 1: Methodology Int. J. Cond. Monit. 1 4653 https://doi.org/10.1784/204764211798303805

[5] Kotila V, Lahdelma S and Ruotsalainen K 2010 Waveletbased Hölder regularity analysis in condition monitoring Computational methods (Integral Methods in Science and Engineering vol 2) ed C Constanda and M E Pérez (Boston, MA: Birkhäuser) pp 233-42 https://doi.org/10.1007/978-0-8176-4897-8_22

[6] Jaffard S 1989 Exposants de Hölder en des points donnés et coéfficients d'ondelettes C.R. Acad. Sci. Paris I 308 79-81

[7] Mallat S, Hwang W L 1992 Singularity detection and processing with wavelets IEEE Trans. Inform. Theory 38 617-43

https://doi.org/10.1109/18.119727

[8] Robertson A N, Farrar C R and Sohn H 2002 Singularity detection for structural health monitoring using Holder exponents Mech. Syst. Signal Process. 17 1163-84 https://doi.org/10.1006/mssp.2002.1569

[9] Miao Q, Huang H-Z and Fan X 2007 Singularity detection in machinery health monitoring using Lipschitz exponent function J. Mech. Sci. Technol. 21 737-44 https://doi.org/10.1007/BF02916351

[10] Kumar H S, Srinivasa P P, Sriram N S and Vijay G S 2013 Rolling element bearing condition classification using Hoelder exponents NMAMIT Annu. Res. J. 3 43-8 https://www.researchgate.net/publication/ 283081913_Rolling_Element_Bearing_Condition_ Classification_Using_Hoelder_Exponents

[11] Venkatakrishnan P, Sangeetha S, Gnanasekaran J S, Vishnukumar M G, Padmanaban A S 2014 Analysis of vibration in gearbox sensor data using Lipschitz exponent (LE) function: a wavelet approach Proc. 3rd Int. Conf. on Advances in Control and Optimization of Dynamical Systems (Kanpur, India) (IFAC Proc. vol 47) pp $1067-71$ https://doi.org/10.3182/20140313-3-IN-3024.00102

[12] Peng Z K, Chu F L and Tse P W 2007 Singularity analysis of the vibration signals by means of wavelet modulus maximal method Mech. Syst. Signal. Process. 21 780-94 https://doi.org/10.1016/j.ymssp.2005.12.005

[13] Liu J, Wang W, Golnaraghi F and Liu K 2008 Wavelet spectrum analysis for bearing fault diagnosis Meas. Sci. Technol. 19015105 https://doi.org/10.1088/0957-0233/19/1/015105

[14] Liu J 2012 Shannon wavelet spectrum analysis on truncated vibration signals for machine incipient fault detection Meas. Sci. Technol. 23055604 https://doi.org/10.1088/0957-0233/23/5/055604

[15] Shakya P, Darpe A K and Kulkarni M S 2013 Vibrationbased fault diagnosis in rolling element bearings: ranking of various time, frequency and time-frequency domain data-based damage identification parameters Int. $J$. Cond. Monit. 3 53-62 https://doi.org/10.1784/204764213808146626

[16] Loutridis S, Trochidis A 2004 Classification of gear faults using Hoelder exponents Mech. Syst. Signal. Process. 18 1009-30 https://doi.org/10.1016/j.ymssp.2004.01.007

[17] Miao Q and Makis V 2005 An application of the modulus maxima distribution in machinery condition monitoring J. Qual. Maint. Eng. 11 375-87 https://doi.org/10.1108/13552510510626990

[18] Sun Q and Tang Y 2002 Singularity analysis using continuous wavelet transform for bearing fault diagnosis Mech. Syst. Signal. Process. 16 1025-41 https://doi.org/10.1006/mssp.2002.1474

[19] Nissilä J 2017 Local regularity analysis with wavelet transform in gear tooth failure detection Manag. Syst. Prod. Eng. 25 176-82 https://doi.org/10.1515/mspe-2017-0026

[20] Briggs W and Henson V E 1995 The DFT An Owners Manual for the Discrete Fourier Transform (Philadelphia, PA: SIAM) p 429

[21] Nissilä J, Lahdelma S and Laurila J 2014 Condition monitoring of the front axle of a load haul dumper with real order derivatives and generalised norms Proc. 11th Int. Conf. on Condition Monitoring and Machinery Failure Prevention Technologies CM2014/MFPT2014 (Manchester) (UK: BINDT)

http://urn.fi/urn:nbn:fi-fe2018091835968

[22] McKechan D J A, Robinson C and Sathyaprakash B S 2010 A tapering window for time-domain templates and simulated signals in the detection of gravitational waves from coalescing compact binaries Class. Quant. Grav. $\mathbf{2 7}$ 084020

https ://doi.org/10.1088/0264-9381/27/8/084020

[23] Lahdelma S and Kotila V 2005 Complex derivative - a new signal processing method Kunnossapito 19 39-46 https://www.researchgate.net/publication/ 266266752_Complex_Derivative_-_A_New_Signal_ Processing_Method

[24] Pinsky M A 2002 Introduction to Fourier Analysis and Wavelets (Pacific Grove, CA: Brooks/Cole) p 376

[25] Zemanian A H 1987 Distribution Theory and Transform Analysis: An Introduction to Generalized Functions with Applications (New York: Dover) p 371

[26] Unser M and Blu T 2000 Fractional splines and wavelets SIAM Rev. 42 43-67 https://doi.org/10.1137/S0036144598349435

[27] Bullen P S 2003 Handbook of Means and Their Inequalities, 2nd edn (Dordrecht: Kluwer)

[28] Lahdelma S, Laurila J, Strackeljan J and Hein R 2011 Separating different vibration sources in complex fault detection Proc. 8th Int. Conf. on Condition Monitoring and Machinery Failure Prevention Technologies CM2011/MFPT2011 (Cardiff) (UK: BINDT) https://www.researchgate.net/publication/ 270048457_Separating_Different_Vibration_Sources_ in_Complex_Fault_Detection

[29] Lahdelma S and Laurila J 2012 Detecting misalignment of a claw clutch using vibration measurements Proc. 9th Int. Conf. on Condition Monitoring and Machinery Failure Prevention Technologies CM2012/MFPT2012 (London) (UK: BINDT) https://www.researchgate.net/publication/ 270048637_Detecting_Misalignment_of_a_Claw_ Clutch_Using_Vibration_Measurements

[30] Laurila J and Lahdelma S 2013 Condition monitoring by means of vibration and sound measurements Proc. 10th Int. Conf. on Condition Monitoring and Machinery 
Failure Prevention Technologies CM2013/MFPT2013 (Kraków) (UK: BINDT)

https://www .researchgate.net/publication/

270048588_Condition_Monitoring_by_Means_of_

Vibration_and_Sound_Measurements

[31] Laurila J and Lahdelma S 2014 Advanced fault diagnosis by means of complex order derivatives Insight, Non-Destr. Test. Cond. Monit. 56 439-42

https://doi.org/10.1784/insi.2014.56.8.439

[32] Holmström L and Koistinen P 2010 Pattern recognition WIREs Comput. Stat. 2 404-13

https://doi.org/10.1002/wics.99 